\title{
In Vivo Diffuse Optical Tomography and Fluorescence Molecular Tomography
}

\author{
Mingze Li, Yonghong Zhang, Jing Bai* \\ Department of Biomedical Engineering, School of Medicine, \\ Tsinghua University, Beijing 100084, China \\ li-mz07@mails.tsinghua.edu.cn \\ zyhbme@mail.tsinghua.edu.cn \\ deabj@tsinghua.edu.cn
}

\begin{abstract}
Diffuse optical tomography (DOT) and fluorescence molecular tomography (FMT) are two attractive imaging techniques for in vivo physiological and psychological research. They have distinct advantages such as non-invasiveness, non-ionizing radiation, high sensitivity and longitudinal monitoring. This paper reviews the key components of DOT and FMT. Light propagation model, mathematical reconstruction algorithm, imaging instrumentation and medical applications are included. Future challenges and perspective on optical tomography are discussed.
\end{abstract}

Keywords: Diffuse Optical Tomography; Fluorescence Molecular Tomography; clinical and preclinical application

\section{INTRODUCTION}

Optical imaging depends on the interaction between light and biological tissue. It uses multiple physical parameters to produce contrast mechanism. Compared with other functional and physiological imaging, optical approaches have distinct advantages such as non-invasiveness, non-ionizing radiation, high sensitivity and longitudinal monitoring. Visualization with light is among the most common practices in today's academic and clinical research [1].

The ability of light to penetrate tissue was first exploited by Bright as early as 1831 [2]. For light in the visible spectrum, penetration depth is limited by strong absorption of hemoglobin and other molecules. In 1977, Jobsis found the 'near-infrared window' between $700 \mathrm{~nm}$ and $1000 \mathrm{~nm}$ [3]. At these wavelengths, absorption by haemoglobin, lipid, and water is minimum. Near-infrared light can penetrate up to several centimeters in biological tissue [4].

Oxy-haemoglobin and deoxy-haemoglobin are two main absorption molecules in the near-infrared spectrum [5]. They are indicators of tissue's blood volume and oxygenation. To monitor these physiological signatures, near-infrared spectroscopy (NIRS) was

*Corresponding author 
developed [6-8]. This technique uses wavelength scanning at a single site. In order to get spatial information, scientists combined multiple NIRS measurements and made the first images of brain [9]. Up to now, many imaging techniques have been developed to produce spatially resolved information. Generally speaking, there are two kinds of techniques, i.e., topography and tomography. The former refers to methods that produce two-dimensional (2D) or planar images of subjects. It has intrinsic inability to quantify or detect deep targets due to the non-linear dependence of light on depth [10]. Diffuse optical tomography (DOT), a three-dimensional (3D) tomographic technique, was developed to overcome the limitation [11].

DOT models the light propagation in tissue by taking the highly scattered photons into account [12]. It illuminates the tissue with near-infrared light from an array of sources and observes the multiply-scattered light using an array of detectors. By means of an inversion scheme, DOT reconstructs the tissue properties (absorption and scattering coefficients) that have close correlation with oxy- and deoxy-haemoglobin concentration blood saturation. Since carcinogenesis features like angiogenesis and hypoxia are often associated with blood saturation, DOT can reveal functional or pathological disorders of living subjects [13].

Traditional DOT is based on absorption and scattering coefficients. The intrinsic contrast mechanism suffers from low sensitivity and lacks specificity to targeted cells. Extrinsically administered molecules are employed to enhance the contrast [14]. These reagents often aggregate in the tumor region while washed out in the normal ones. Among them, Indocyanine Green (ICG) is the only NIR agent approved by the US Food and Drug Administration (FDA) for human use [15].

Fluorescence molecular tomography (FMT) is another strategy to improve the contrast and detection specificity. It was first put forward by Vasilis et al. in 2002 [16]. It shares tomographic principles of DOT, but simultaneously uses optical properties and fluorescence measurements for accurate 3D reconstruction of exogenous probes, such as green fluorescent protein (GFP), red fluorescent protein (RFP), cyanine dyes (Cy5.5, Cy7) and nano-particles [17]. These injected fluorophores accumulate preferentially in diseased tissue. This specificity distribution as well as their different decay properties could be useful to localize tumors [18].

FMT has unique features such as repeatable excitation and emission, in vivo multitarget labeling and visualization of specific physiological activity [19]. In addition, the use of fluorophore can also have access to information like $\mathrm{pO}_{2}, \mathrm{pH}$ and intracellular calcium concentration [20]. In recent years, FMT has aroused increasing interest among scientists and clinicians [19,21-23].

This work reviews the key components of DOT and FMT. First, we describe the fundamental principle of DOT, including light propagation model, mathematical algorithm and imaging instrumentation. The current clinical application in optical mammography, infant brain imaging and detection of other tissues are followed. Unlike DOT, most of in vivo studies using FMT are conducted on small animals. We present basic theories of FMT in a separate section with the emphasis on its distinctive features and preclinical application. In the end of this article, future challenges and perspective on optical tomography are discussed. 


\section{FUNDAMENTAL PRINCIPLE OF DOT}

\subsection{Light Propagation Model}

When near-infrared light travels through biological tissue, it will undergo reflection, diffraction, absorption, scattering, etc [24]. Modeling light propagation through tissues is critical for making quantitative optical imaging a feasible endeavor [25, 26].

There are many different models to describe light propagation within tissue [27-30]. Generally, they can be classified into two categories: deterministic and stochastic.

Deterministic models use Maxwell equation to well interpret the interaction between light and medium taking into account scattering, absorption and reflection. But it is too complex to get its analytic solution in practice. In most biological tissues, the effect of scattering dominates. Radiative transfer equation (RTE) is usually chosen to treat photons as elastic particles after ignoring any wave effects [31]. RTE is a conservation formulation describing the change of energy radiance due to changes in energy flow. Its analytical solution is usually scarce and exists only in certain simple and homogeneous cases [32]. When geometries of tissue and the distribution of optical properties are complicated, diffusion equation is favorable. Diffusion approximation is based on the assumption that scattering dominates over absorption and regions of interest are far from sources and detectors [30]. This is generally the case in bulk tissues, but it breaks down in regions near the source, the surface, internal boundaries, as well as in anisotropic and high absorbing or low scattering tissues. In these situations, the higher-order spherical harmonics approximation [33] or RTE-based models $[34,35]$ may be required.

Stochastic models simulate the trajectories of individual photon that either escapes from the boundary or is absorbed by the tissue. By tracking a sufficient number of photons, physical quantities such as diffuse reflectance can be estimated [36]. The procedures are conceptually simpler to implement and rely on fewer assumptions, but at the expense of computational time. Among them, Monte Carlo (MC) is the most commonly used method [28]. It incorporates Poisson error into the model naturally and simulates the photon migration in both the diffusive and non-diffusive domains [37-39]. In fact, MC is often regarded as the 'golden standard' in diffuse optics [40]. Another statistical approach is the Random walk theory, where photon transport is modeled as a series of steps on a discrete cubic lattice. It is particularly suited to time-domain measurements, and has been used, for example, to quantify the optical properties of a breast tumor [41] and to simulate diffusion in brain extracellular space [42]. More details can be found in [12].

\subsection{Reconstruction Algorithm}

Photons do not follow straight paths as X-ray does, so the standard back projection in computer tomography (CT) is no longer accurate for image reconstruction. Instead, the model is dictated by the above-mentioned Maxwell equation, radiative transport equation, or the diffusion approximation. Two problems are involved: the forward problem and the inverse problem [43].

The forward problem uses the light transport model to predict the distribution of light in the object under examination. This stage generates a sensitivity matrix (the 
Jacobian) that relates the measurements to the internal optical properties. Since scatter is dominant, the forward problem becomes a series of integrals over the entire volume. Arridge first derived the analytical solution using Green's function for different geometries [44] and various modification such as Kirchhoff approximation [45] and regularization theories[46] were proposed. Finite element method (FEM) [12, 47], finite difference method (FDM) [48-50], finite volume method (FVM) [49, 51, 52] and boundary element method (BEM) [53-55] are employed to discrete the continuous partial differential equations (PDE). Among them, FEM is most widely used, because it can represent the inhomogeneous distribution of optical properties in an arbitrary geometry $[30,56]$.

In the inverse problem, the sensitivity matrix is inverted and the spatial distribution of optical parameters is reconstructed according to the boundary measurements. Since each measurement is sensitive to the whole volume, the inverse problem is often illposed. Up to now, many reconstructive techniques have been proposed [30, 57]. For example, Hielscher and Klose proposed an iterative image reconstruction model (MOBIIR) based on the equation of radiative transfer [58, 59]. Davis et al. compared the Tikhonov approach with a modified Levenberg-Marquardt formulation [60]. Schweiger et al. attempted Gauss-Newton method [61], and Konovalov et al. used algebraic reconstruction with post-processing [62]. Generally, these approaches can be categorized into linear reconstruction and non-linear reconstruction [63]. Linear problems use difference data between two neighboring states to quantify images of measured changes, rather than absolute quantitative ones [30]. Nonlinear reconstruction establishes an objective function and repeatedly narrows the gap between predicted data and measurements to update the reconstructed variables [64]. More details of reconstruction algorithm can be found in reference [65].

\subsection{Imaging Instrumentation}

With regard to excitation-detection manner, there are three types of optical imaging systems: continuous-wave (CW), time-domain (TD), and frequency-domain (FD).

$\mathrm{CW}$ mode requires a source that either emits at a constant intensity or is modulated at a low (a few $\mathrm{kHz}$ ) frequency [63]. The transmitted light is collected to resolve the attenuation. The major advantages of $\mathrm{CW}$ system include its compactness and economical hardware as well as optimum signal-to-noise performance. Philips Research Laboratories evaluated a breast tomography system based on CW measurements[66]. Schmitz et al. used a CW DOT system, named DYNOT (NIRx Medical technologies, NY) to reveal cyclic hemodynamic changes[67]. Other companies like Imaging Diagnostic Systems Inc. and Advanced Research Technologies Inc. once developed diffuse optical imagers. Nowadays, CW systems have been intensively used in optical tomography [68-71].

TD technology uses a short pulse of light (100 fs $100 \mathrm{ps})$ to illuminate the medium. The time-of-flight and amplitude of photons are recorded by high-speed electronic instrumentation, such as streak cameras, fast avalanche photodiodes and gated optical image intensifiers [24]. Temporal distribution of photons can be described with 
temporal point spread function (TPSF), which extends over several nanoseconds after travelling through several centimeters. For instance, a 32-channel time-resolved system was developed based on time-correlated single-photon counting (TCSPC) technology [67]. Some research was conducted on a custom-made dual-wavelength $(670 \mathrm{~nm}, 785 \mathrm{~nm})$ time-domain optical mammogram instrument [72]. Eda et al. built a 64-channel timeresolved optical tomographic imaging system [73]. Other studies using TD systems can be found in [74-76].

FD technology uses modulated light of a modulated intensity at $100 \mathrm{MHz}$ to $1 \mathrm{GHz}$ frequency. Amplitude attenuation and phase shift of diffuse photon density wave (DPDW) are measured by sensitive detectors such as a gain-modulated ICCD [77]. Data obtained at multiple frequencies improve its performance over CW mode and is equivalent to TD data via the inverse Fourier Transform. During the mid-1990s, two companies, Carl Zeiss and Siemens have developed two breast imaging systems based on FD measurements [40]. In 2001, McBride et al. reported a FD system for breast imaging using five optical wavelengths and 16 photomultiplier tubes [78]. Recently, Orlova et al. have invented an experimental multicolor FD DOT system to visualize neoplastic of breast tissue [79].

It is noted that each mode has its pros and cons. CW system is compact and inexpensive. But it is somewhat difficult to distinguish absorption with scatter. TD and FD setups suffer from low signal-to-noise ratio and complex structures, though their contrast and resolution are higher than CW mode. For more details refer to [40].

\section{MEDICAL APPLICATION OF DOT}

\subsection{Optical Mammography}

Breast cancer is among the leading causes of death for women all over the world [80]. The survival chance of breast cancer drops from a rate of about $95 \%$ when the lesion is about $0.5 \mathrm{~cm}$ in size to a rate of $75 \%$ when the cancer is treated at a size of about $2.5 \mathrm{~cm}$ [81]. Early detection and treatment is of vital importance for decreasing mortality.

$\mathrm{X}$-ray mammography is the routine method for mass screening of the population. However, relatively large lesion is required to produce detectable contrast. X-ray also causes ionizing radiation and its diagnosis is not satisfactory in premenopausal women. On the other hand, magnetic resonance imaging (MRI) and ultrasound techniques are technically demanding and not suitable for routine inspection [82].

Optical mammography utilizes non-ionizing, low-power near-infrared light to scan the female breast [83]. It measures wavelength-dependent tissue optical absorption coefficient, which in turn provides the access to blood dynamics, total hemoglobin concentration (THC) and tissue blood oxygen saturation $\left(\mathrm{StO}_{2}\right)$ [84]. Since tumors are often associated with increased vascularization [85], the contrast in growing tumors is physiologically plausible for optical imaging. The noninvasive nature of NIR and its potential of high specificity are also attractive for human breast imaging.

Optical techniques for imaging the breast can be traced back to the late 1920's [86]. Cutler presented the first clinical results using transillumination as an aid in the diagnosis of breast lesions. In the past two decades, optical techniques received 
renewed attention owing to the development of accurate mathematic models and reconstruction strategies [87]. Especially in recent years, diffuse optical tomography has been intensively applied in 3D breast imaging [82, 88-90].

Generally, optical mammography features either a compressed or an uncompressed system, as shown in Figure 1. The former measures optical properties using parallel plate geometry. The breast is compressed either between two parallel arrays of sources and detectors, or between two plates over which individual sources and detectors are scanned in a rectilinear manner. Vasilis and his colleagues at the Massachusetts General Hospital and Harvard Medical School first used this configuration to obtain images of a 70 -year-old patient with a $0.8 \mathrm{~cm}$ infiltrating ductal carcinoma [93]. ICG was used as the contrast agent and optical results were validated by simultaneous MRI images. Later, they integrated optical tomography into a breast X-ray tomosynthesis system and measured 18 patients, aged 49 to 79 , all of whom were scheduled for biopsy for suspicious findings [91]. Optical images were reconstructed for both benign and malignant lesions, revealing optical contrast close to the location of the lesions shown in the corresponding X-ray images. They also studied the spatio-temporal imaging of hemoglobin in the compressed breast with this setup and observed the global return of blood following compression [94].

(a)

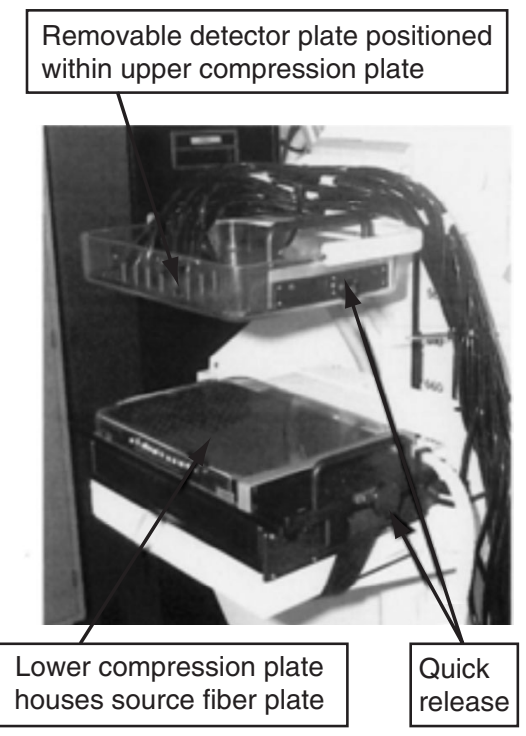

(b)

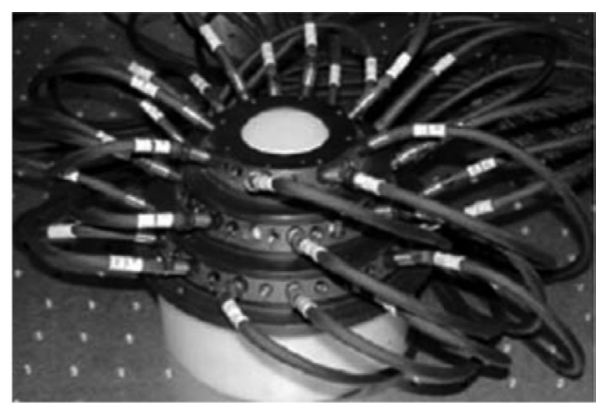

Figure 1. (a) Structure of compression plates designed for coregistered X-ray and optical breast imager [91].

(b) Arrangement of source-detector fiber bundles attached to three interconnecting rings [92]. 
Researchers have also developed a three-dimensional diffuse optical tomography system in the parallel-plate compression geometry. They studied three cases of invasive ductal carcinoma, ductal and lobular carcinoma in situ and fibro adenoma. The data demonstrated that malignant lesions had a twofold average increase in optical parameters, while the benign tumors did not exhibit significance in the tumor-to-normal ratios of any parameter. Figure 2 shows the DOT result of a 53-year-old woman with a 2.2-cm invasive ductal carcinoma in her right breast comparing with MRI images [83].

It is considered that compression may cause some physiological changes or potential reduction in the blood volume [95, 96]. Uncompressed geometry was explored and developed fast in recent years. As early as 1997, Pogue et al. built a frequency domain optical imaging device for breast cancer detection [97]. The imaging chamber was composed of 16 source and 16 detectors located around a twodimensional ring with the pendulous breast stabilized naturally within it. With this system, the distribution of absorption and reduced scattering coefficients of a patient with a $3.4 \mathrm{~cm}$ fibro adenoma in the upper central region of her breast was displayed [98]. They also quantified typical values of hemoglobin concentration, oxygen saturation, water fraction, scattering power, and scattering amplitude within the breast tissue, and conducted a systematic study of the menstrual variations in these parameters [99]. Later, they integrated MRI into the system and imaged the breast tissue of 11 normal female subjects. Higher water and blood signals were found in fibro-glandular fraction than that in adipose tissue [100].

Using a DOT system to image uncompressed breast, Jiang et al. conducted a study to differentiate cysts from solid tumors. The pilot results showed that solid breast tumors demonstrated higher absorption and scattering related to the normal tissue while cysts had lower absorption and scattering coefficients compared with the surrounding normal tissue [101]. Yates et al. imaged uncompressed breast using 32-channel timeresolved system composed of a conical fiber holder in the form of three connecting rings. The breast was placed in a hemispherical cup surrounded by sources and detectors, and the remaining space was filled with a fluid with tissue-like optical properties [102]. One of their experiments was conducted with three healthy volunteers and twenty-one patients. Seventeen cases of lesion were successfully detected [92]. They also used this system to monitor the changes of optical properties after laser treatment of a fibro adenoma in breast tissue. Images of the absorbing and scattering coefficients revealed the expected response consistent with corresponding ultrasound examinations [103]. A series of clinical three-dimensional optical images have been presented, showing that hypervascularization associated with tumors provides high contrast due to the increased absorption by hemoglobin [104].

Some commercial systems are currently available for breast imaging such as DYNOT (NIRX Inc., NY, USA) [105], CTLM (Imaging Diagnostic Systems Inc., FL, USA) [106], and SoftScan (ART Inc., Quebec, Canada) [107-109] . The first two work in CW mode while the SoftScan is based on time-resolved measurements. SoftScan uses two plates to perform compression, while CTLM uses uncompressed 
(a)

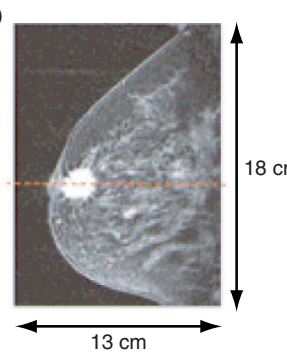

(b)

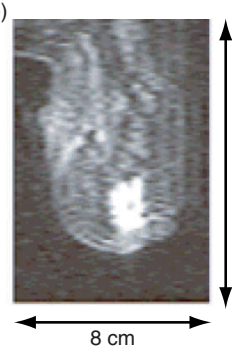

(c)

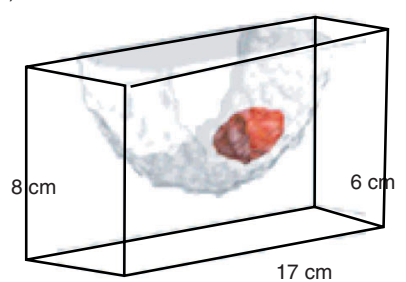

(d) Relative total hemoglobin concentration

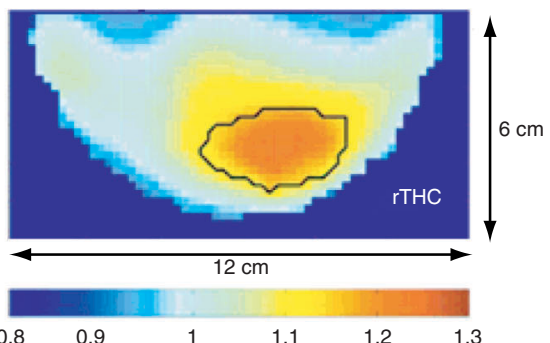

(f) Relative oxygenated hemoglobin
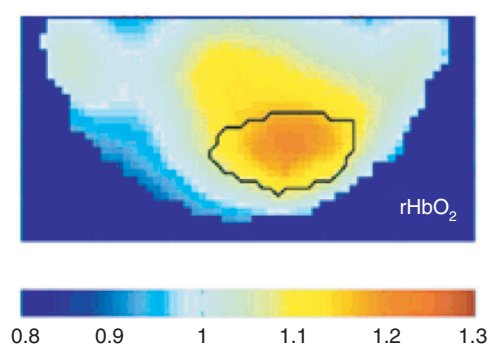

(h) Relative tissue scattering
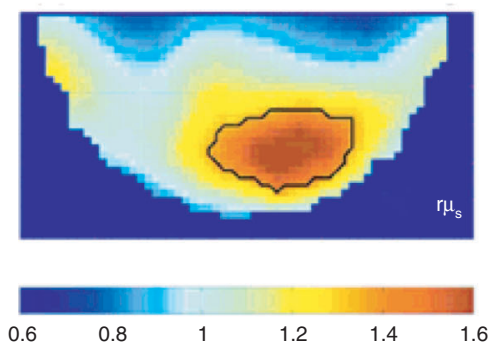

(e) Relative blood oxygen saturation

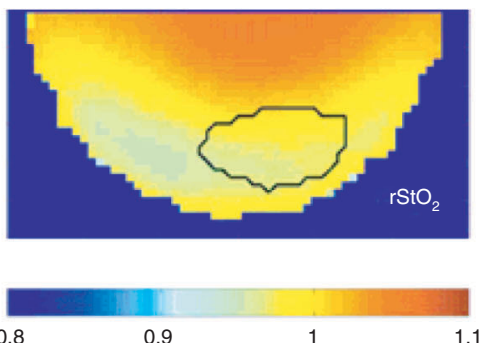

(g) Relative deoxygenated hemoglobin

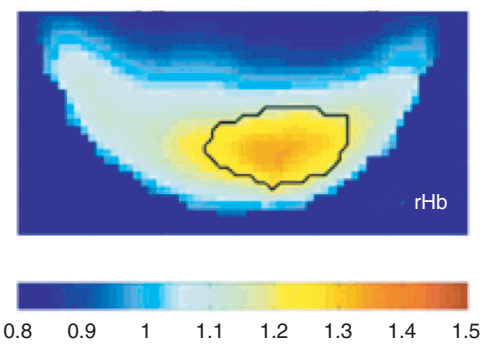

(i) Optical index

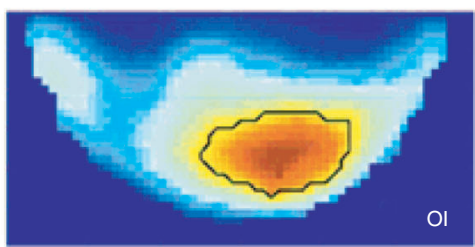

$\begin{array}{llllllll}0.6 & 0.8 & 1 & 1.2 & 1.4 & 1.6 & 1.8 & 2\end{array}$

Figure 2. MRI and DOT images of a 53-year-old woman with a 2.2-cm invasive ductal carcinoma in her right breast [83]. (a) The sagittal dynamic-contrastenhanced (DCE) -MRI showing the tumor center. (b) The axial DCE MRI slice along the red horizontal line in (a), oriented in caudal-cranial view. 
chamber. DYNOT system has exchangeable heads with various geometries including circular geometry for the limbs, folding hemisphere for the breast, helmetlike device for the head, and various two-dimensional fiber array designs for nearly planar geometries [110].

\subsection{Infant Brain Imaging}

Optical brain imaging has undergone 30 years of intense development [111]. It is portable and less sensitive to motion artifact (compared with functional MRI). It can obtain repeated quantitative regional information and longitudinal monitoring of brain function (unfeasible for CT). Furthermore, it avoids the radioactive exposure that may cause injury in the neonate or premature infant (superior to PET) [112]. Therefore, it is particularly suitable for infant brain.

Optical diagnosis includes spectroscopy, 2D topography and 3D tomography. In the beginning, NIRS was used to study infant cerebral hemodynamic and neural activation [113]. Later, optical topography was employed to produce 2D images of activated regions on the surface of the brain [114-118]. To identify changes occurring in deeper tissues, tomographic methods have been studied to produce $3 \mathrm{D}$ volumetric images of the whole neonatal brain [119-123].

As early as 1985, Arridge et al. pointed out that NIR trans-illumination could be used to visualize and measure the oxygenation state of brain and muscle in newborn infants [124]. In 2000, Benaron et al. demonstrated the first tomographic images of infant brain [125]. They measured the flight times of photons between points on the head circumference with a headband of 34 source-detector pairs. Images of an infant undergoing heart-lung bypass and another infant with hypoxic-ischemic injury were successively showcased and compared with CT, ultrasound and MRI images [125, 126].

Hebden et al. used a 32-channel time-resolved instrument (named MONSTIR) as a continuous bedside monitor to obtain functional images of premature infants' brains [127]. Sources and detectors are coupled to the infant head by a custom-made foamlined plastic helmet [128]. Although the positions of the bundles on the helmet are recorded using a 3D digitizing arm immediately before or after the clinical

\section{Figure 2. (Continued)}

Enhancement of gadolinium uptake in MRI indicates the malignancy. (c) The tumor region (in red) determined based on optical data with the guidance of MRI and the breast outline in 3D space. DOT images of (d) relative total hemoglobin concentration rTHC, (e) relative blood oxygen saturation $\mathrm{rStO} 2$, (f) relative oxygenated hemoglobin concentration $\mathrm{rHbO}$, (g) relative deoxygenated hemoglobin concentration $\mathrm{rHb}$, (h) relative tissue scattering $\mathrm{r} \mu_{\mathrm{s}}$ at $786 \mathrm{~nm}$ and (i) optical index are shown in caudal-cranial view with a black solid line indicating the region identified as tumor using a region-growing algorithm. High tumor-to-normal contrast in rTHC, $\mathrm{rHbO} 2, \mathrm{rHb}, \mathrm{r}_{\mathrm{s}}$ and $\mathrm{OI}$ are visible within the region. 
measurement, these are not always sufficiently accurate for absolute imaging given the natural displacement during the scan. To overcome this problem, scientists inserted a 'reference' subject (fluid-filled balloon, fluid-filled latex shell or compressible head phantom) into the helmet for a difference imaging $[119,129,130]$. They compared the acquired data with those obtained from the homogenous reference and found that regional cerebral blood volume $(\mathrm{rCBV})$ and regional tissue oxygen saturation $\left(\mathrm{rSTO}_{2}\right)$ from healthy infants were symmetrical, while those from intraventricular hemorrhage (IVH) were asymmetrical with a much greater light absorption on the side of hemorrhage [131].

Optical tomography can detect not only static brain injury, but also changes in brain oxygenation due to changes in inspired oxygen and carbon dioxide in ventilated infants. Imaging by MONSTIR system was reported on a severely brain-injured 38-week-old female infant who had suffered a global hypoxia-ischemic insult following uterine rupture [132]. Three dimensional absorption images of brain in response to the increase in ventilated $\mathrm{CO}_{2}$ were reconstructed. Gao et al. also studied hemodynamic changes in response to the alterations of the ventilation settings on two preterm infant brains [75].

DOT has also been applied in imaging passive motor-evoked responses in premature babies. Gibson et al. conducted optical tomography during raising and lowering an infant's arm. Their reconstructed results showed good agreement of changes in optical property with the expected anatomical position of the contra lateral motor cortex, as shown in Figure 3 [131].

DOT has not been used as widely in infant brain as optical topography and spectroscopy [132-134]. This is partly due to the complexity of instrumentation and difficulties in taking measurements from premature babies. Moreover, light transmission across the whole head is challenging due to its serious attenuation with depth [135].

\subsection{Other Tissues}

In addition to the above typical clinical applications, optical tomography has been employed in the 3D imaging of high scattering media such as human forearm and low scattering region like finger joint.

The forearm muscle has been studied by optical imaging since 1994 [136]. In 2000, Graber et al. used a CW optical tomography system to explore the real-time response of the forearm vasculature to rhythmic contraction of antagonistic striated muscle groups [137]. Hillman et al. also evaluated adult forearm using their 32-channel TD system [138]. Zhao et al. used an NIR DOT system composed of time-correlated single-photon-counting channels to obtain in vivo images of human lower legs and forearm. Their images showed increases in blood volume and oxyhemoglobin concentration in the arteries and hypoxia in the corresponding muscles [139].

Rheumatoid arthritis (RA) is a chronic, progressive, inflammatory disease that primarily attacks peripheral joints and surrounding tendons and ligaments. As early as 1998, Klose et al. developed an NIR scanning system to collect amplitude and phase delay of photon density waves in frequency domain [140]. Xu et al. investigated the 


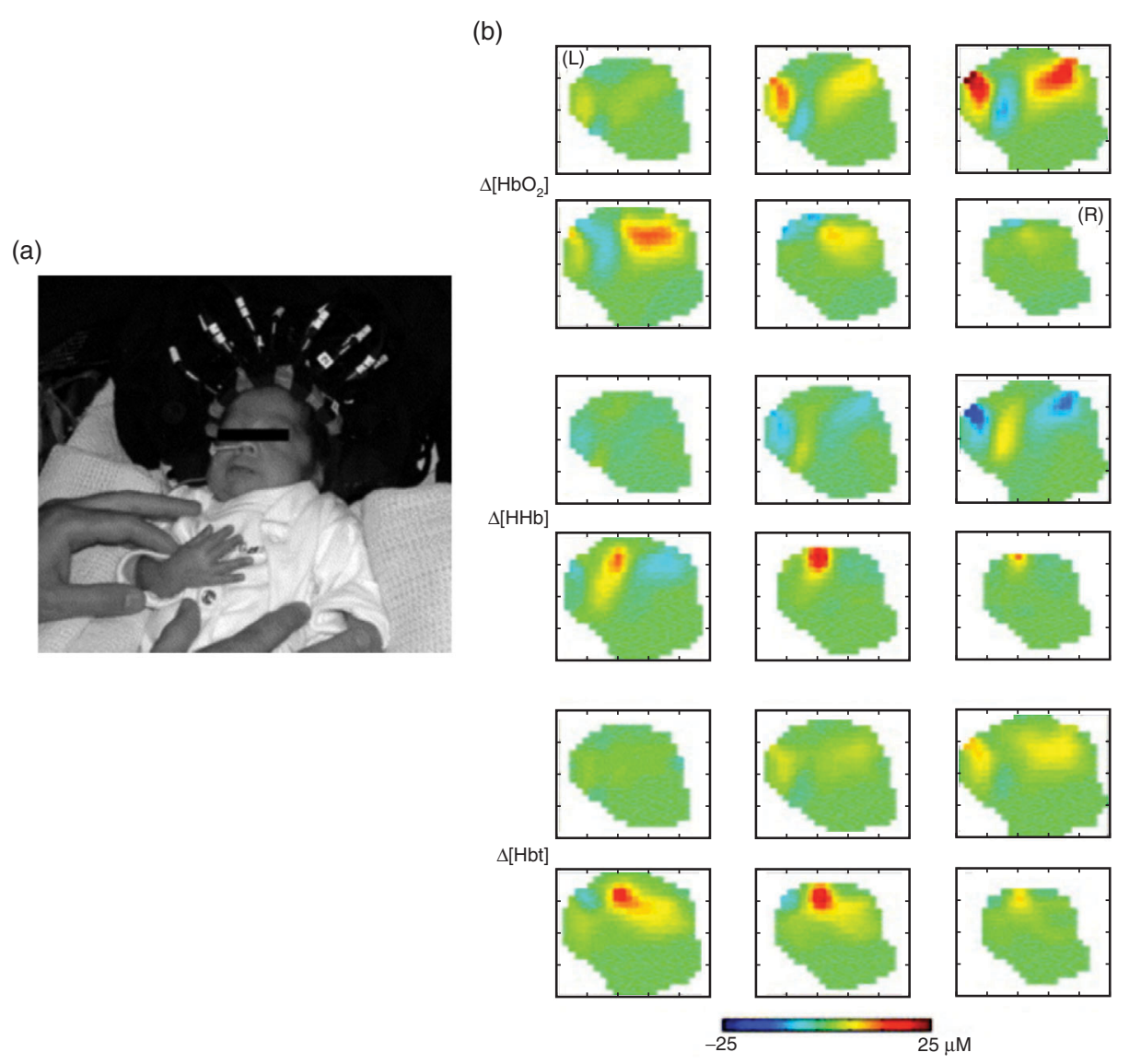

Figure 3. (a) Activating the right arm of a neonate wearing a helmet with optical tomography connectors. (b) Images of $\Delta[\mathrm{HbO} 2], \Delta[\mathrm{HHb}]$, and $\Delta[\mathrm{HbT}]$ during left motor activity, showing increases in $\Delta[\mathrm{HHb}]$ and $\Delta[\mathrm{HbT}]$ near the estimated position of the motor cortex [131].

in vitro and in vivo bones and joints with a Clemson multi-channel diffuse optical imager based on CW measurements [70]. In 2005, Hielscher reported a sagittal laser optical tomography for rheumatoid finger joints [141] and was clinically evaluated by Scheel et al. a year later [142]. Using dual-wavelength tomography and MOBIIR software, Hielscher et al. proposed a dynamic optical tomography system and observed differences of hemodynamic effect in finger joints between healthy volunteers and RA patients [143]. Recently, new diagnostic strategies such as photoacoustic tomography [144] and X-ray guided optical tomography [145] have been explored for RA detection. 


\section{FUNDAMENTAL PRINCIPLE OF FMT}

\subsection{Theoretical Models}

The interaction of light with tissue in FMT is similar to that in DOT. The main difference falls in the number of equations. Fluorescence involves the following two processes: (1) fluorescent probes absorb excitation energy and change from the ground to the excited state, and (2) the excited fluorophore returns to the ground state with a characteristic time constant as well as the emission of photons with a longer wavelength. Two parameters are commonly used to describe the fluorescence phenomena: the fluorescence lifetime and Stoke shift.

Predicting photon paths in real high scattering and anisotropic tissues is extremely complex. Statistical methods based on Monte Carlo are valid to model fluorescent photon trajectories [146-149]. However, in most cases, two coupled RTE or DE approximations are preferable [81, 150-158]. One of the equations describes the excitation plane wave from the external light source to the medium while the other refers to the fluorescence emission from the fluorophore marker to the detector. Other mathematical formulations such as Monte Carlo with diffusion model [159, 160], hybrid transport and diffusion model $[35,161,162]$ and telegrapher equation (TE) based model [163] have also been reported.

\subsection{Reconstruction Algorithm}

The inverse problem in FMT is to reconstruct the concentration distribution or lifetime of fluorescent probes. Many effective algorithms have been proposed, including the gradient-based optimization technique [164-168], penalty/modified barrier function (PMBF) method [169-171], Born-type approximation techniques [172,173], Landweber iteration [174], Newton's or Newton-type optimization methods [175], Bayesian nonlinear least squares approaches [176], adaptive finite-element-based method [177], Tikhonov regularization method [178], the matrix-free algorithm [179], to name just a few.

FMT features an ill-posed inverse problem with certain challenges. One challenge is the optical heterogeneity of biological tissue [180-183]. The fluorescence intensity recorded at tissue boundary is a comprehensive effect of the fluorescence distribution and the tissue absorption and scattering distribution. However, it is difficult to determine tissue's optical properties. Most of the current approaches assume a homogeneous background with known absorption and scattering parameters. On the other hand, normalized Born ratios [172], video reflectometry measurements [25] and DOT guided FMT $[150,184]$ are proposed to deal with the heterogeneity.

FMT is also greatly affected by the excitation leakage [185-187], system noise [188] as well as the nonspecific background autofluorescence [189]. Strategies such as using interference filters[186] or gradient index (GRIN) lenses [187], data pre-processing with subtraction $[190,191]$ or pre-filtration schemes $[192,193]$, as well as multispectral imaging [194] have been attempted to remove these influences.

FMT suffers from a large number of unknowns and a relatively limited number of measurements, which essentially causes under-determined equations. A promising solution is to use a priori information to guide the reconstruction $[195,196]$. Typically, two types of prior information are available. One type is the localization information of 
a certain fluorophore based on its chemical and physical properties. The other is the internal edge structure information from another imaging modality such as CT [197] , MRI [198], PET [199], and ultrasound [200].

\subsection{Imaging Systems}

There are three types of FMT instrumentation: CW, TD and FD. FMT prototype for small animal was first developed by Ntziachristos et al. [16]. In 2003, Graves et al. developed a constant-wave sub-millimeter resolution imaging system that enabled planar imaging as well as fluorescence tomography [173]. Fluorescence measurement of referenced ac intensity and phase shift in response to point illumination measurement geometry was conducted using a homodyned intensified charge-coupled device system [201]. In 2005, Patwardhan et al. reported a TD FMT system for imaging the kinetics of probe distributions through the whole body of small animals [202]. Other researches on TD system can be found in [203-207].

The past two decades have witnessed the great structural improvement in the FMT system. For example, the earlier systems were mainly based on fiber coupling and imaging chambers with matching fluids [172]. Later, the cumbersome system was replaced with the flying spot illumination and charge coupled device (CCD)-based detection for multi-view boundary measurements [173, 208]. In 2007, a non-contact free-space fluorescence tomography system of full angle geometry was reported [209]. Such imaging strategies eliminate the need for individual fibers in contact with the highly scattering volume. In addition, noncontact measurements from diffuse media could facilitate the use of large detector arrays at multiple angles that are well-suited for tomography applications. Recently, real-time continuous detection becomes a new trend, and is expected to realize full-angle dynamic observation superior to the traditional stepby-step mode [210]. Figure 4 shows the schematic rendering of such a system.

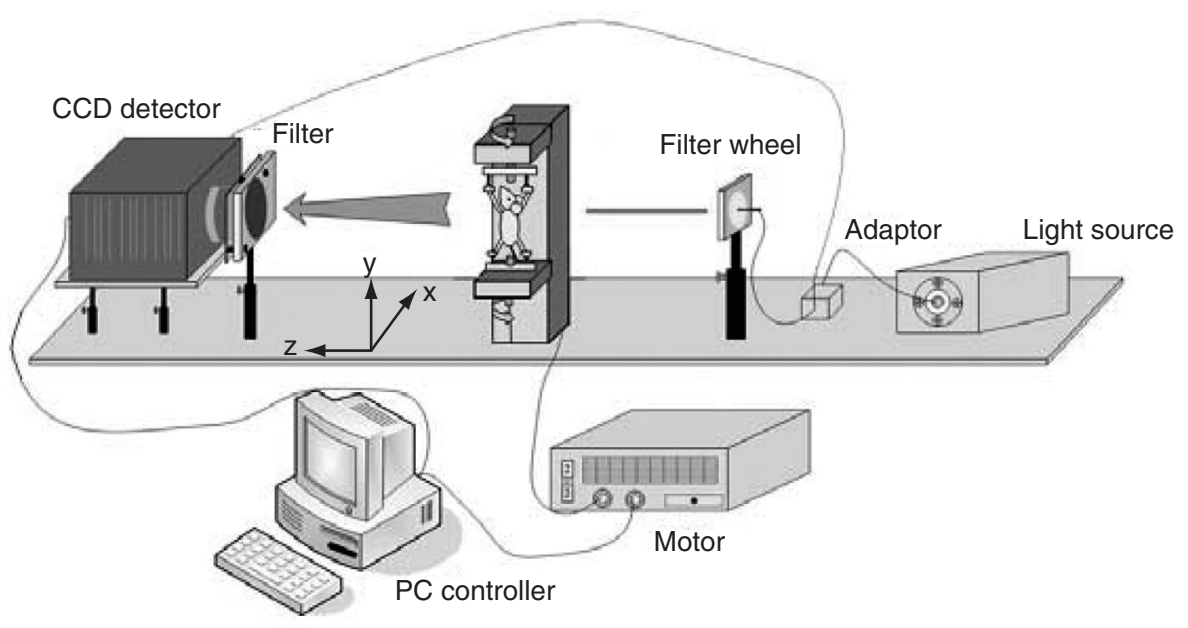

Figure 4. Non-contact full-angle continuous FMT system. 
Currently, there are some commercial systems for in vivo small animal fluorescence imaging, such as IVIS ${ }^{\circ}$ Spectrum (Xenogen Corporation), FMT 2500LX (VisEn Medical Corporation), Maestro ${ }^{\mathrm{TM}}$ (Cambridge Research \& Instrumentation Incorporation), Pearl ${ }^{\mathrm{TM}}$ Imager (Li-COR Biosciences), eXplore Optix ${ }^{\circledR}$ Series (General Electric Healthcare Corporation), etc. Among them, IVIS ${ }^{\circledR}$ Spectrum and FMT 2500LX can perform threedimensional tomography in some applications. For more details see reference [211].

\subsection{Preclinical Application}

Unlike DOT, FMT has not reached a state of maturity which allows routine clinical practice in human. Most of preclinical studies have been conducted on small animals or tissue phantoms.

As early as 2002, Vasilis et al. used a prototype FMT imager to obtain threedimensional in vivo images of a protease in orthopic gliomas [16]. They found a correlation between matrix metalloproteinase, cathepsinsB and the HT-1080 tumor burden [212]. In terms of tumor responses to treatment, they studied antitumor treatment with an annexin V-Cy5.5 conjugate [213]. With the same system they quantified tumor therapeutic modulation with an anti-vascular endothelial growth factor (anti-VEGF) antibody drug [214]. After the appearance of non-contact configuration, 3D in-vivo images of GFP-expressing T-cells in mice were reported [215]. FMT has been so far successfully applied in pulmonary inflammation [216], lung cancer [217], neurological disorders [218], cardiovascular diseases [219, 220], immunologic diseases [221], etc. Human breast phantom has been extensively studied using fluorescence-enhanced optical tomography [77, 171, 192, 201, 222-227].

Combination of FMT with other modalities is another growing application. For example, Davis et al. implanted U-251 human gliomas into nude mice and completed MRI and FMT acquisition after administration of a VEGF-targeted NIR fluorophore. Tissue structural information obtained from standard and contrast enhanced T1weighted images was used to spatially constrain the FMT reconstruction [228]. Gliomas response to chemotherapy was also explored by combined FMT-MRI system in the living mouse brain [229]. Hybrid FMT-CT imaging is another example which has been applied to detect inflammation in murine atherosclerotic plaques [230], and amyloid- $\beta$ plaques in a murine Alzheimer's disease model [218]. Figure 5 compares the ex vivo results with in vivo FMT imaging for a 13-month-old C57B/6 control mouse (first row), a 17-month-old APP23 tg mouse (Second row), and a 26-month-old APP23 tg mouse (third row) [218].

The process of drug development is a long, high-risky and costly endeavor with an average of 10 15 years and \$1.9 billion for a new approved drug [231]. Imaging methods, such as positron emission tomography (PET) [232], MRI [233] and bioluminescence [234], have been employed to enhance the speed of preclinical study for novel anti-cancer agents. In 2007, three-dimensional FMT was adopted to explore the effect of a vascular endothelial growth factor (VEGF) blockade on angiogenesis [235]. Scientists have designed experiments to evaluate the treatment of 


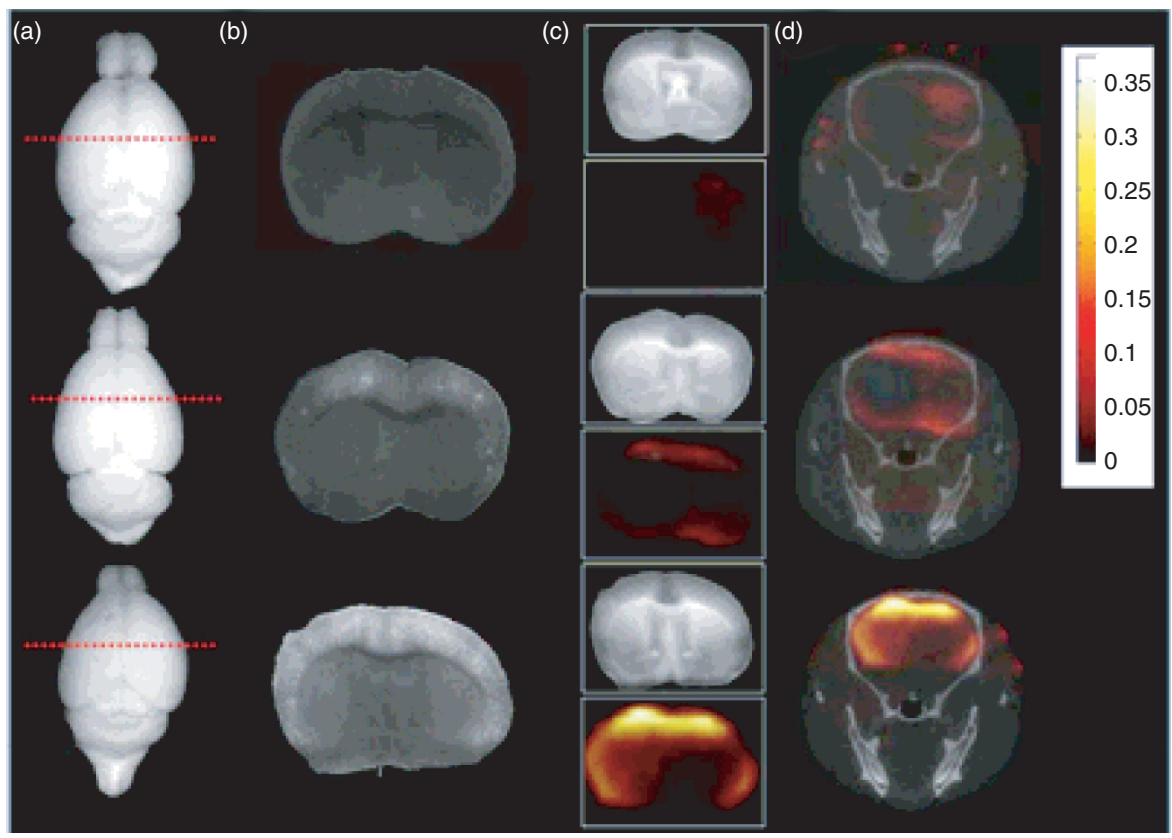

Figure 5. (a) Full brain images in the excitation channel using a planar reflectance imaging system. The red dotted line denotes the approximate location corresponding to the slice shown in subsequent columns. (b) Planar reflectance images of normalized fluorescence from a single slice. (c) Planar images at the excitation wavelength (top) and FMT reconstructions overlaid on normalized planar fluorescence images (bottom). (d) In vivo multi-modal FMT reconstructions for a slice corresponding to the same location as the ex vivo images, overlaid on a representative CT slice. All FMT reconstructions are scaled to the same colorbar.

the anti-VEGF antibody. Fifty-eight CT26 colon tumor-bearing mice were imaged after intravenous administration of long-circulating near-infrared fluorescent bloodpool agents. Figure 6 shows FMT imaging in three cohorts of mice: animals receiving no treatment (normal), animals receiving saline (PBS), and animals receiving treatment (Avastin) [235].

As mentioned earlier, specific fluorophores are vital for the application of FMT. To optimize the parameters in labeling NIR fluorescent dye, Qian et al. inspected the stability of cypate-protein conjugate in blood serum and its distribution in small animals under various labeling conditions [236]. Preclinical application of FMT is now rapidly growing [211, 237-239]. 


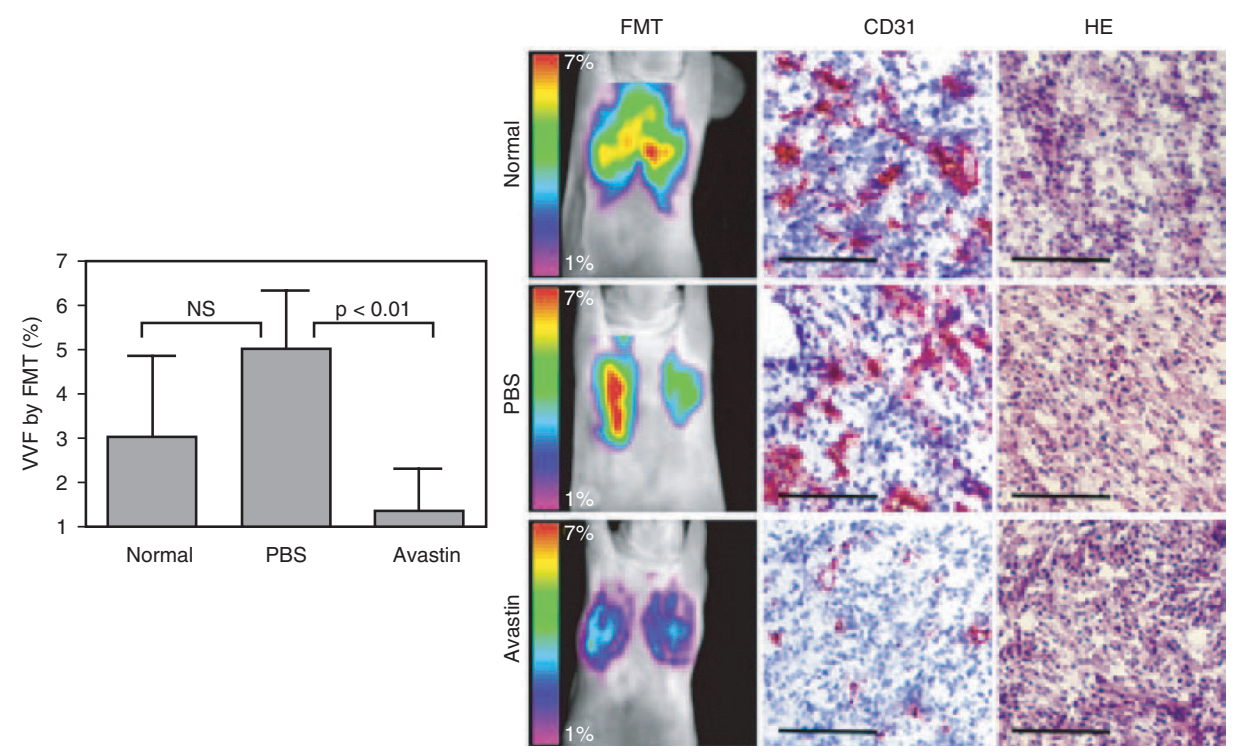

Figure 6. In vivo angiogenic data. Scale bar $=100 \mu \mathrm{m}$. NS $=$ not significant. Error bars $=$ standard deviation. Vascular volume fraction $(\mathrm{VVF})$ for treatment group was lower than control and baseline groups. Fluorescence tomographic images were validated with immunohistochemical staining including CD31 and hematoxylineosin (HE).

\section{CONCLUSION}

After decades of development, DOT has emerged from a research concept to a practical, working clinical tool [240]. Though FMT is not as mature as DOT, it is an evolving field that has already achieved major advances in human breast phantom and small animal imaging. Currently, the major difficulties may be in the improvement of quantitation, spatial resolution, as well as the identification of more target-specific biomarkers [135]. Most of the light propagation models are based on simplified assumptions which may lead to inaccuracies in the imaging. Advances in theory and reconstruction algorithms are needed to facilitate the development of more sophisticated models. Additionally, more efficient computational technology and imaging instrumentation are in demand to collect large data sets. It is hoped that optical tomography may become a promising alternative to the existing imaging technologies.

Multi-modality imaging, which combines two or more different imaging techniques into one context, is an emerging field in both clinical research and small animal imaging [241-244]. As modern medical and diagnostic technology develops, comprehensive information provided by different imaging modalities may be needed. In the past several years, DOT-ultrasound [245], DOT-MRI [246], DOT-CT [247], FMT-MRI 
[248], FMT-CT [249, 250], FMT-PET [251] and FMT-ultrasound [252] have been extensively studied. Although few multimodality systems have been clinically applied to date, there are encouraging signs that a new generation of multi-modality imaging will emerge and find wider application in the near future.

\section{ACKNOWLEDGEMENT}

This work is supported by the National Natural Science Foundation of China under Grant No. 60831003, 30930092, 30872633; the Tsinghua-Yue-Yuen Medical Science Foundation; the National Basic Research Program of China under Grant No.2006CB705700; the National High-Tech Research and Development Program of China 863 under Grant No. 2006AA020803.

\section{REFERENCES}

[1] Balas, C., Review of biomedical optical imaging-a powerful, non-invasive, non-ionizing technology for improving in vivo diagnosis, Measurement Science \& Technology, 2009, 20(10).

[2] Bright, R, Diseases of the brain and nervous system, Longman, London, UK, 1831.

[3] Jobsis, FF, Noninvasive, infrared monitoring of cerebral and myocardial oxygen sufficiency and circulatory parameters, Science, 1977, 198(4323), 1264.

[4] Wang, LV, Wu, HI and Masters, BR, Biomedical Optics, Principles and Imaging, Journal of Biomedical Optics, 2008, 13, 049902.

[5] Crespi, F, Near-Infrared Spectroscopy (NIRS): A Non-Invasive In Vivo Methodology for Analysis of Brain Vascular and Metabolic Activities in Real Time in Rodents, Current Vascular Pharmacology, 2007, 5(4), 305-321.

[6] Mancini, DM, Bolinger, L, Li, H, Kendrick, K, Chance, B and Wilson, JR, Validation of near-infrared spectroscopy in humans, Journal of Applied Physiology, 1994, 77(6), 2740.

[7] Brazy, JE, Near-infrared spectroscopy, Clinics in perinatology, 1991, 18(3), 519.

[8] Wyatt, JS, Cope, M, Delpy, DT, Richardson, CE, Edwards, AD, Wray, S and Reynolds, EO, Quantitation of cerebral blood volume in human infants by near-infrared spectroscopy, Journal of Applied Physiology, 1990, 68(3), 1086.

[9] Gratton, G., Corballis, P. M., Cho, E. H., Fabiani, M. and Hood, D. C., ed., Shades of Gray-Matter Noninvasive Optical-Images of Human Brain Responses During Visual-Stimulation: 34th Annual Meeting of the Society-for-Psychophysiological-Research, Soc Psychophysiol Res, Atlanta, Ga, 1994, 505-509.

[10] Fujii, M. and Nakayama, K., Signal filtering algorithm for depth-selective diffuse optical topography, Physics in Medicine and Biology, 2009, 54(6), 1419-1433.

[11] Hebden, J. C., Optical tomography: Development of a new medical imaging modality, Medical Physics, 1998, 440, 79-90.

[12] Arridge, S. R. and Hebden, J. C., Optical imaging in medicine .2. Modelling and reconstruction, Physics in Medicine and Biology, 1997, 42(5), 841-853.

[13] Klose, A. D. and Hielscher, A. H., Functional imaging of small tissue volumes with diffuse optical tomography, Medical Imaging 2006: Physiology, Function, and Structure from Medical Images Pts 1 and 2, 2006, 6143, 1431.

[14] Bremer, C, Ntziachristos, V and Weissleder, R, Optical-based molecular imaging: contrast agents and potential medical applications, European Radiology, 2003, 13(2), 231-243.

[15] Alt nog lu, EI, Russin, TJ, Kaiser, JM, Barth, BM, Eklund, PC, Kester, M and Adair, JH, Near-infrared emitting fluorophore-doped calcium phosphate nanoparticles for in vivo imaging of human breast cancer, ACS nano, 2008, 2(10), 2075-2084. 
[16] Ntziachristos, Vasilis, Tung, Ching-Hsuan, Bremer, Christoph and Weissleder, Ralph, Fluorescence molecular tomography resolves protease activity in vivo, Nature Medicine, 2002, 8(7), 757-760.

[17] Sevick-Muraca, E. M. and Rasmussen, J. C., Molecular imaging with optics: primer and case for nearinfrared fluorescence techniques in personalized medicine, Journal of Biomedical Optics, 2008, 13(4).

[18] Bloch, S, Lesage, F, McIntosh, L, Gandjbakhche, A, Liang, K and Achilefu, S, Whole-body fluorescence lifetime imaging of a tumor-targeted near-infrared molecular probe in mice, Journal of Biomedical Optics, 2005, 10, 054003.

[19] Ripoll, J., Meyer, H. and Garofalakis, A., In vivo optical tomography: From diffusion to ballistic, Optical Materials, 2009, 31(7), 1082-1085.

[20] Corlu, A., Choe, R., Durduran, T., Rosen, M. A., Schweiger, M., Arridge, S. R., Schnall, M. D. and Yodh, A. G., Three-dimensional in vivo fluorescence diffuse optical tomography of breast cancer in humans, Optics Express, 2007, 15(11), 6696-6716.

[21] Schulz, R. B., Ale, A., Sarantopoulos, A., Freyer, M., Sohngen, R., Zientkowska, M. and Ntziachristos, V., Hybrid fluorescence tomography/X-ray tomography improves reconstruction quality, Proceedings of the SPIE - The International Society for Optical Engineering, 2009, 7370, 4.

[22] Lin, Y. T., Yan, H., Nalcioglu, O. and Gulsen, G., Quantitative fluorescence tomography with functional and structural a priori information, Applied Optics, 2009, 48(7), 1328-1336.

[23] Deliolanis, N. C., Dunham, J., Wurdinger, T., Figueiredo, J. L., Tannous, B. A. and Ntziachristos, V., In-vivo imaging of murine tumors using complete-angle projection fluorescence molecular tomography, Journal of Biomedical Optics, 2009, 14(3), 3.

[24] Hebden, J. C., Arridge, S. R. and Delpy, D. T., Optical imaging in medicine .1. Experimental techniques, Physics in Medicine and Biology, 1997, 42(5), 825-840.

[25] Comsa, D. C., Farrell, T. J. and Patterson, M. S., Quantitative fluorescence imaging of point-like sources in small animals, Physics in Medicine and Biology, 2008, 53(20), 5797-5814.

[26] Kolehmainen, V., Schweiger, M., Nissila, I., Tarvainen, T., Arridge, S. R. and Kaipio, J. P., Approximation errors and model reduction in three-dimensional diffuse optical tomography, Journal of the Optical Society of America a-Optics Image Science and Vision, 2009, 26(10), 2257-2268.

[27] Yuan, Z., Hu, X. H. and Jiang, H. B., A higher order diffusion model for three-dimensional photon migration and image reconstruction in optical tomography, Physics in Medicine and Biology, 2009, 54(1), 65-88.

[28] Liu, K, Tian, J, Yang, W, Qin, CH, Xu, M and Liu, D, Applications of Monte Carlo Method in Simulating Diffuse Optical Imaging, Journal of Software, 2009, 20(5), 1216-1225.

[29] Karbeyaz, E. and Rappaport, C. M., Modal-based tomographic imaging from far-zone observations, Journal of the Optical Society of America a-Optics Image Science and Vision, 2009, 26(1), 19-29.

[30] Dehghani, H., Srinivasan, S., Pogue, B. W. and Gibson, A., Numerical modelling and image reconstruction in diffuse optical tomography, Philosophical Transactions of the Royal Society a-Mathematical Physical and Engineering Sciences, 2009, 367(1900), 3073-3093.

[31] Tarvainen, T., Vauhkonen, M., Kolehmainen, V., Kaipio, J. P. and Arridge, S. R., Utilizing the radiative transfer equation in optical tomography, Piers 2008 Cambridge, Proceedings, 2008, 730-735.

[32] Arridge, S. R. and Schweiger, M., Image reconstruction in optical tomography, Philosophical Transactions of the Royal Society B-Biological Sciences, 1997, 352(1354), 717-726.

[33] Wright, S., Schweiger, M. and Arridge, S. R., ed., Reconstruction in optical tomography using the $P-N$ approximations: 5th International Conference on Inverse Problems in Engineering, Iop Publishing Ltd, Cambridge, ENGLAND, 2005, 79-86.

[34] Dehghani, H., Delpy, D. T. and Arridge, S. R., Photon migration in non-scattering tissue and the effects on image reconstruction, Physics in Medicine and Biology, 1999, 44(12), 2897-2906.

[35] Tarvainen, T., Vauhkonen, M., Kolehmainen, V., Arridge, S. R. and Kaipio, J. P., Coupled radiative transfer equation and diffusion approximation model for photon migration in turbid medium with lowscattering and non-scattering regions, Physics in Medicine and Biology, 2005, 50(20), 4913-4930.

[36] Rjasanow, S, Stochastic numerics for the Boltzmann equation, 2008, 385, 219. 
[37] Zhao, H. J., Zhou, X. Q., Liang, J. L. and Zhang, S. Q., Fast Monte Carlo inversion for extracting the optical properties of tubular tissues, Chinese Optics Letters, 2008, 6(12), 935-937.

[38] Parys, B., Papalexandris, M. V. and Cornet, A., Assessment of femtosecond temporal gating for imaging in biological medium by Monte Carlo simulations, Iee Proceedings-Vision Image and Signal Processing, 2006, 153(2), 181-184.

[39] Boas, D. A., Culver, J. P., Stott, J. J. and Dunn, A. K., Three dimensional Monte Carlo code for photon migration through complex heterogeneous media including the adult human head, Optics Express, 2002, 10(3), 159-170.

[40] Gibson, A. P., Hebden, J. C. and Arridge, S. R., Recent advances in diffuse optical imaging, Physics in Medicine and Biology, 2005, 50(4), R1-R43.

[41] Chernomordik, V., Hattery, D. W., Grosenick, D., Wabnitz, H., Rinneberg, H., Moesta, K. T., Schlag, P. M. and Gandjbakhche, A., Quantification of optical properties of a breast tumor using random walk theory, Journal of Biomedical Optics, 2002, 7(1), 80-87.

[42] Jin, S, Zador, Z and Verkman, AS, Random-Walk Model of Diffusion in Three Dimensions in Brain Extracellular Space: Comparison with Microfiberoptic Photobleaching Measurements, Biophysical Journal, 2008, 95(4), 1785-1794.

[43] Arridge, S. R. and Schotland, J. C., Optical tomography: forward and inverse problems, Inverse Problems, 2009, 25(12).

[44] Arridge, S. R., Cope, M. and Delpy, D. T., The Theoretical Basis for the Determination of Optical Pathlengths in Tissue-Temporal and Frequency-Analysis, Physics in Medicine and Biology, 1992, 37(7), 1531-1560.

[45] Ripoll, J., Ntziachristos, V., Culver, J., Yodh, A. G. and Nieto-Vesperinas, M., The Kirchhoff Approximation in diffusive media with arbitrary geometry, Photon Migration, Optical Coherence Tomography, and Microscopy, 2001, 2(31), 134-140.

[46] Sassaroli, A. and Martelli, F., Perturbation theory for the diffusion equation by use of the moments of the generalized temporal point-spread function. II. Continuous-wave results, Journal of the Optical Society of America a-Optics Image Science and Vision, 2006, 23(9), 2119-2131.

[47] Arridge, S. R., Schweiger, M., Hiraoka, M. and Delpy, D. T., A Finite-Element Approach for Modeling Photon Transport in Tissue, Medical Physics, 1993, 20(2), 299-309.

[48] Hielscher, A. H., Alcouffe, R. E. and Barbour, R. L., Comparison of finite-difference transport and diffusion calculations for photon migration in homogeneous and heterogeneous tissues, Physics in Medicine and Biology, 1998, 43(5), 1285-1302.

[49] Klose, A. D. and Hielscher, A. H., Optical tomography with the equation of radiative transfer, International Journal of Numerical Methods for Heat \& Fluid Flow, 2008, 18(3-4), 443-464.

[50] Eppstein, M. J., Dougherty, D. E., Hawrysz, D. J. and Sevick-Muraca, E. M., Three-dimensional optical tomography, Optical Tomography and Spectroscopy of Tissue Iii, Proceedings Of, 1999, 3597, 97-105.

[51] Ren, K., Abdoulaev, G. S., Bal, G. and Hielscher, A. H., Algorithm for solving the equation of radiative transfer in the frequency domain, Optics Letters, 2004, 29(6), 578-580.

[52] Ren, K., Bal, G. and Hielscher, A. H., Frequency domain optical tomography based on the equation of radiative transfer, Siam Journal on Scientific Computing, 2006, 28(4), 1463-1489.

[53] Srinivasan, S., Pogue, B. W., Carpenter, C., Yalavarthy, P. K. and Paulsen, K., A boundary element approach for image-guided near-infrared absorption and scatter estimation, Medical Physics, 2007 , 34(11), 4545-4557.

[54] Sikora, J., Zacharopoulos, A., Douiri, A., Schweiger, M., Horesh, L., Arridge, S. R. and Ripoll, J., Diffuse photon propagation in multilayered geometries, Physics in Medicine and Biology, 2006, 51(3), 497-516.

[55] Fedele, F., Eppstein, M. J., Laible, J. P., Godavarty, A. and Sevick-Muraca, E. M., Fluorescence photon migration by the boundary element method, Journal of Computational Physics, 2005, 210(1), 109-132. 
[56] Cong, A. X. and Wang, G., A finite-element-based reconstruction method for 3D fluorescence tomography, Optics Express, 2005, 13(24), 9847-9857.

[57] Schweiger, M., Gibson, A. and Arridge, S. R., Computational aspects of diffuse optical tomography, Computing in Science \& Engineering, 2003, 5(6), 33-41.

[58] Hielscher, AH, ed., Model-based iterative image reconstruction for photon migration tomography, 1997, 106.

[59] Klose, $\mathrm{AD}$ and Hielscher, $\mathrm{AH}$, Iterative reconstruction scheme for optical tomography based on the equation of radiative transfer, Medical Physics, 1999, 26, 1698.

[60] Davis, S. C., Dehghani, H., Yalavarthy, P. K., Pogue, B. W. and Paulsen, K. D., Comparing two regularization techniques for diffuse optical tomography - art. no. 64340X, Optical Tomography and Spectroscopy of Tissue VII, 2007, 6434, 4340.

[61] Schweiger, M., Arridge, S. R. and Nissila, I., Gauss-Newton method for image reconstruction in diffuse optical tomography, Physics in Medicine and Biology, 2005, 50(10), 2365-2386.

[62] Konovalov, A. B., Vlasov, V. V., Mogilenskikh, D. V., Kravtsenyuk, O. V. and Lyubimov, V. V., Algebraic reconstruction and postprocessing in one-step diffuse optical tomography, Quantum Electronics, 2008, 38(6), 588-596.

[63] Hiescher, A. H., Recent advances in optical tomographic imaging, 2007 Pacific Rim Conference on Lasers and Electro-Optics, Vols 1-4, 2007, 877-878.

[64] Boverman, G., Miller, E. L. and Boas, D. A., Linear and nonlinear reconstruction for diffuse optical tomography in an inhomogeneous background, Computational Imaging Ii, 2004, 5299, 10-21.

[65] Arridge, S. R., ed., Linear and non-linear methods in optical tomography: Biomedical Topical Meeting, Optical Soc America, Miami Beach, Fl, 2000, 495-497.

[66] Colak, S. B., van der Mark, M. B., Hooft, G. W., Hoogenraad, J. H., van der Linden, E. S. and Kuijpers, F. A., Clinical optical tomography and NIR spectroscopy for breast cancer detection, IEEE Journal of Selected Topics in Quantum Electronics, 1999, 5(4), 1143-1158.

[67] Schmitz, CH, L cker, M, Lasker, JM, Hielscher, AH and Barbour, RL, Instrumentation for fast functional optical tomography, Review of Scientific Instruments, 2002, 73, 429.

[68] Su, L. Z., Shan, H., Liu, H. L. and Klibanov, M. V., Reconstruction method with data from a multiplesite continuous-wave source for three-dimensional optical tomography, Journal of the Optical Society of America a-Optics Image Science and Vision, 2006, 23(10), 2388-2395.

[69] Li, A., Zhang, Q., Culver, J. P., Miller, E. L. and Boas, D. A., Reconstructing chromosphere concentration images directly by continuous-wave diffuse optical tomography, Optics Letters, 2004, 29(3), 256-258.

[70] Xu, Y., Iftimia, N., Jiang, H. B., Key, L. L. and Bolster, M. B., Imaging of in vitro and in vivo bones and joints with continuous-wave diffuse optical tomography, Optics Express, 2001, 8(7), 447-451.

[71] Jiang, H. B., Xu, Y. and Iftimia, N., Experimental three-dimensional optical image reconstruction of heterogeneous turbid media from continuous-wave data, Optics Express, 2000, 7(5), 204-209.

[72] Grosenick, D., Wabnitz, H., Moesta, K. T., Mucke, J., Moller, M., Stroszczynski, C., Stossel, J., Wassermann, B., Schlag, P. M. and Rinneberg, H., Concentration and oxygen saturation of haemoglobin of 50 breast tumours determined by time-domain optical mammography, Physics in Medicine and Biology, 2004, 49(7), 1165-1181.

[73] Eda, H., Oda, I., Ito, Y., Wada, Y., Oikawa, Y., Tsunazawa, Y., Takada, M., Tsuchiya, Y., Yamashita, Y., Oda, M., Sassaroli, A., Yamada, Y. and Tamura, M., Multichannel time-resolved optical tomographic imaging system, Review of Scientific Instruments, 1999, 70(9), 3595-3602.

[74] Ma, Y. W., Gong, S. R., Gao, F., Yang, F., Zhao, H. J. and Jiang, J. Y., Improvement of performances in time-domain breast DOT by the aid of image segmentation, Multimodal Biomedical Imaging Iii, $2008,6850,500$.

[75] Gao, F., Ma, Y. W., Yang, F., Zhao, H. J., Jiang, J. Y., Kusaka, T., Ueno, M. and Yamada, Y. K., Imaging hemodynamic changes in preterm infant brains with two-dimensional diffuse optical tomography, Advanced Biomedical and Clinical Diagnostic Systems Vi, 2008, 6848, 8481. 
[76] Zhao, H. J., Gao, F., Tanikawa, Y., Homma, K. and Yamada, Y., Time-resolved diffuse optical tomographic imaging for the provision of both anatomical and functional information about biological tissue, Applied Optics, 2005, 44(10), 1905-1916.

[77] Godavarty, A., Eppstein, M. J., Zhang, C. Y., Theru, S., Thompson, A. B., Gurfinkel, M. and SevickMuraca, E. M., Fluorescence-enhanced optical imaging in large tissue volumes using a gainmodulated ICCD camera, Physics in Medicine and Biology, 2003, 48(12), 1701-1720.

[78] McBride, T. O., Pogue, B. W., Jiang, S., Osterberg, U. L. and Paulsen, K. D., A parallel-detection frequency-domain near-infrared tomography system for hemoglobin imaging of the breast in vivo, Review of Scientific Instruments, 2001, 72(3), 1817-1824.

[79] Orlova, A. G., Turchin, I. V., Plehanov, V. I., Shakhova, N. M., Fiks, I. I., Kleshnin, M. I., Konuchenko, N. Y. and Kamensky, V. A., Frequency-domain diffuse optical tomography with single source-detector pair for breast cancer detection, Laser Physics Letters, 2008, 5(4), 321-327.

[80] Tang, J, Rangayyan, RM, Xu, J, Naqa, IE and Yang, Y, Computer-aided detection and diagnosis of breast cancer with mammography: Recent advances, IEEE Transactions on Information Technology in Biomedicine, 2009, 13(2), 236-251.

[81] Roy, R., Godavarty, A. and Sevick-Muraca, E. M., Fluorescence-enhanced optical tomography of a large tissue phantom using point illumination geometries, Journal of Biomedical Optics, 2006, 11(4).

[82] Karellas, A. and Vedantham, S., Breast cancer imaging: A perspective for the next decade, Medical Physics, 2008, 35(11), 4878-4897.

[83] Choe, R., Konecky, S. D., Corlu, A., Lee, K., Durduran, T., Busch, D. R., Pathak, S., Czerniecki, B. J., Tchou, J., Fraker, D. L., DeMichele, A., Chance, B., Arridge, S. R., Schweiger, M., Culver, J. P., Schnall, M. D., Putt, M. E., Rosen, M. A. and Yodh, A. G., Differentiation of benign and malignant breast tumors by in-vivo three-dimensional parallel-plate diffuse optical tomography, Journal of Biomedical Optics, 2009, 14(2).

[84] Shah, N, Cerussi, AE, Jakubowski, D, Hsiang, D, Butler, J and Tromberg, BJ, Spatial variations in optical and physiological properties of healthy breast tissue, Journal of Biomedical Optics, 2004, 9, 534.

[85] Wessels, J. T., Busse, A. C., Mahrt, J., Dullin, C., Grabbe, E. and Mueller, G. A., In vivo imaging in experimental preclinical tumor research - A review, Cytometry Part A, 2007, 71A(8), 542-549.

[86] Catler, M, Transillumination as an aid in the diagnosis of breast lesions. With special reference to its value in cases of bleeding nipple, Surg. Gynecol. Obstet, 1929, 48, 721-729.

[87] Yodh, A and Chance, B, Spectroscopy and imaging with diffusing light, Physics Today, 1995, 48(3), 34-41.

[88] Tromberg, B. J., Pogue, B. W., Paulsen, K. D., Yodh, A. G., Boas, D. A. and Cerussi, A. E., Assessing the future of diffuse optical imaging technologies for breast cancer management, Medical Physics, 2008, 35(6), 2443-2451.

[89] Edelhauser, G. and Funovics, M., Breast Cancer Treatment in the Era of Molecular Imaging, Breast Care, 2008, 3(6), 409-414.

[90] Leff, DR, Warren, OJ, Enfield, LC, Gibson, A, Athanasiou, T, Patten, DK, Hebden, J, Yang, GZ and Darzi, A, Diffuse optical imaging of the healthy and diseased breast: A systematic review, Breast Cancer Research and Treatment, 2008, 108(1), 9-22.

[91] Zhang, Q., Brukilacchio, T. J., Li, A., Stott, J. J., Chaves, T., Hillman, E., Wu, T., Chorlton, M., Rafferty, E., Moore, R. H., Kopans, D. B. and Boas, D. A., Coregistered tomographic X-ray and optical breast imaging: initial results, Journal of Biomedical Optics, 2005, 10(2), 024033-1 024033-9.

[92] Yates, T, Hebden, JC, Gibson, A, Everdell, N, Arridge, SR and Douek, M, Optical tomography of the breast using a multi-channel time-resolved imager, Physics in Medicine and Biology, 2005, 50, 2503-2517.

[93] Ntziachristos, V., Yodh, A. G., Schnall, M. and Chance, B., Concurrent MRI and diffuse optical tomography of breast after indocyanine green enhancement, Proceedings of the National Academy of Sciences of the United States of America, 2000, 97(6), 2767-2772. 
[94] Boverman, G, Fang, Q, Carp, SA, Miller, EL, Brooks, DH, Selb, J, Moore, RH, Kopans, DB and Boas, DA, Spatio-temporal imaging of the hemoglobin in the compressed breast with DOT, Physics in Medicine and Biology, 2007, 52, 3619-3641.

[95] Carp, SA, Selb, J, Fang, Q, Moore, R, Kopans, DB, Rafferty, E and Boas, DA, Dynamic functional and mechanical response of breast tissue to compression, Optics Express, 2008, 16(20), 16064-16078.

[96] Carp, SA, Kauffman, T, Fang, Q, Rafferty, E, Moore, R, Kopans, D and Boas, D, Compressioninduced changes in the physiological state of the breast as observed through frequency domain photon migration measurements, Journal of Biomedical Optics, 2006, 11, 064016.

[97] Pogue, B, Testorf, M, McBride, T, Osterberg, U and Paulsen, K, Instrumentation and design of a frequency-domain diffuse optical tomography imager for breast cancer detection, Optics Express, 1997, 1(13), 391-403.

[98] Pogue, BW, Poplack, SP, McBride, TO, Wells, WA, Osterman, KS, Osterberg, UL and Paulsen, KD, Quantitative Hemoglobin Tomography with Diffuse Near-Infrared Spectroscopy: Pilot Results in the Breast1, Radiology, 2001, 218(1), 261.

[99] Pogue, B. W., Jiang, S. D., Dehghani, H., Kogel, C., Soho, S., Srinivasan, S., Song, X. M., Tosteson, T. D., Poplack, S. P. and Paulsen, K. D., Characterization of hemoglobin, water, and NIR scattering in breast tissue: Analysis of intersubject variability and menstrual cycle changes, Journal of Biomedical Optics, 2004, 9(3), 541-552.

[100] Brooksby, B., Pogue, B. W., Jiang, S. D., Dehghani, H., Srinivasan, S., Kogel, C., Tosteson, T. D., Weaver, J., Poplack, S. P. and Paulsen, K. D., Imaging breast adipose and fibroglandular tissue molecular signatures by using hybrid MRI-guided near-infrared spectral tomography, Proceedings of the National Academy of Sciences of the United States of America, 2006, 103(23), 8828-8833.

[101] Gu, X. J., Zhang, Q. Z., Bartlett, M., Schutz, L., Fajardo, L. L. and Jiang, H. B., Differentiation of cysts from solid tumors in the breast with diffuse optical tomography, Academic Radiology, 2004, 11(1), 53-60.

[102] Yates, T. D., Hebden, J. C., Gibson, A. P., Enfield, L., Everdell, N. L., Arridge, S. R. and Delpy, D. T., Time-resolved optical mammography using a liquid coupled interface, Journal of Biomedical Optics, 2005, 10(5), 10.

[103] Hebden, J. C., Yates, T. D., Gibson, A., Everdell, N., Arridge, S. R., Chicken, D. W., Douek, M. and Keshtgar, M. R. S., Monitoring recovery after laser surgery of the breast with optical tomography: a case study, Applied Optics, 2005, 44(10), 1898-1904.

[104] Enfield, LC, Gibson, AP, Everdell, NL, Delpy, DT, Schweiger, M, Arridge, SR, Richardson, C, Keshtgar, M, Douek, M and Hebden, JC, Three-dimensional time-resolved optical mammography of the uncompressed breast, Applied Optics, 2007, 46(17), 3628-3638.

[105] Schmitz, CH, Klemer, DP, Hardin, R, Katz, MS, Pei, Y, Graber, HL, Levin, MB, Levina, RD, Franco, NA and Solomon, WB, Design and implementation of dynamic near-infrared optical tomographic imaging instrumentation for simultaneous dual-breast measurements, Applied Optics, 2005, 44(11), $2140-2153$.

[106] Floery, D, Helbich, TH, Riedl, CC, Jaromi, S, Weber, M, Leodolter, S and Fuchsjaeger, MH, Characterization of benign and malignant breast lesions with computed tomography laser mammography (CTLM): initial experience, Investigative Radiology, 2005, 40(6), 328.

[107] Intes, X.et.al, Time-domain optical mammography SoftScan: initial results, Academic Radiology, 2005, (12), 934-47.

[108] Khayat, M, Ichalalene, Z, Mincu, N, Leblond, F, Guilman, O and Djeziri, S, ed., Optical tomography as adjunct to $x$-ray mammography: methods and results, $2007,64310 \mathrm{~F}$.

[109] Mincu, N, Djeziri, S, Ichalalène, Z, Leblond, F and Khayat, M, ed., Sensitivity and repeatability of diffuse optical tomography: towards breast cancer neoadjuvant treatment monitoring, 2007, 64341D.

[110] Schmitz, CH, Graber, HL, Barbour, RL, Lasker, JM, Hielscher, AH and Pei, Y, ed., A real-time system for dynamic optical tomography, 2005. 
[111] Hillman, E. M. C., Optical brain imaging in vivo: techniques and applications from animal to man, Journal of Biomedical Optics, 2007, 12(5).

[112] Hintz, SR, CHEONG, WAIF, VAN HOUTEN, JP, STEVENSON, DK and BENARON, DA, Bedside imaging of intracranial hemorrhage in the neonate using light: comparison with ultrasound, computed tomography, and magnetic resonance imaging, Pediatric research, 1999, 45(1), 54.

[113] Cope, M, The application of near infrared spectroscopy to non invasive monitoring of cerebral oxygenation in the newborn infant, Department of Medical Physics and Bioengineering, University College London, 1991, 214-9.

[114] Watanabe, H, Homae, F, Nakano, T and Taga, G, Functional activation in diverse regions of the developing brain of human infants, Neuroimage, 2008, 43(2), 346-357.

[115] Taga, G, Konishi, Y, Maki, A, Tachibana, T, Fujiwara, M and Koizumi, H, Spontaneous oscillation of oxy-and deoxy-hemoglobin changes with a phase difference throughout the occipital cortex of newborn infants observed using non-invasive optical topography, Neuroscience letters, 2000, 282(1-2), 101-104.

[116] Hintz, SR, Benaron, DA, Siegel, AM, Zourabian, A, Stevenson, DK and Boas, DA, Bedside functional imaging of the premature infant brain during passive motor activation, Journal of Perinatal Medicine, 2001, 29(4), 335-343.

[117] Taga, G and Asakawa, K, Selectivity and localization of cortical response to auditory and visual stimulation in awake infants aged 2 to 4 months, Neuroimage, 2007, 36(4), 1246-1252.

[118] Taga, G, Asakawa, K, Maki, A, Konishi, Y and Koizumi, H, Brain imaging in awake infants by nearinfrared optical topography, Proceedings of the National Academy of Sciences, 2003, 100(19), 10722.

[119] Austin, T., Gibson, A. P., Branco, G., Yusof, R. M., Arridge, S. R., Meek, J. H., Wyatt, J. S., Delpy, D. T. and Hebden, J. C., Three dimensional optical imaging of blood volume and oxygenation in the neonatal brain, Neuroimage, 2006, 31(4), 1426-1433.

[120] Austin, T., Gibson, A. P., Everdell, N. L., Herden, J. C., Schweiger, M., Arridge, S. R., Meek, J. H. and Wyatt, J. S., Three dimensional optical imaging of neonatal brain activation, Pediatric research, 2005, $58(2), 16$.

[121] Hebden, J. C., Gibson, A., Austin, T., Yusof, R. M., Everdell, N., Delpy, D. T., Arridge, S. R., Meek, J. H. and Wyatt, J. S., Imaging changes in blood volume and oxygenation in the newborn infant brain using three-dimensional optical tomography, Physics in Medicine and Biology, 2004, 49(7), $1117-1130$.

[122] Yusof, R. M., Hebden, J. C., Gibson, A., Everdell, N., Austin, T., Meek, J. H., Arridge, S. R., Wyatt, J. S. and Delpy, D. T., Validation of the use of homogenous reference phantoms for optical tomography of the neonatal brain, Optical Tomography and Spectroscopy of Tissue V, 2003, 4955, 6-11.

[123] Gibson, A., Yusof, R. M., Dehghani, H., Riley, J., Everdell, N., Richards, R., Hebden, J. C., Schweiger, M., Arridge, S. R. and Delpy, D. T., Optical tomography of a realistic neonatal head phantom, Applied Optics, 2003, 42(16), 3109-3116.

[124] Arridge, S. R., Cope, M., Vanderzee, P., Hillson, P. J. and Delphy, D. T., Near-infrared transillumination as a method of visualization and measurement of the oxygenation state of brain and muscle in newborn-infants, Journal of Photographic Science, 1985, 33(6), 227-227.

[125] Benaron, DA, Hintz, SR, Villringer, A, Boas, D, Kleinschmidt, A, Frahm, J, Hirth, C, Obrig, H, van Houten, JC and Kermit, EL, Noninvasive functional imaging of human brain using light, Journal of Cerebral Blood Flow and Metabolism, 2000, 20(3), 469-477.

[126] Hintz, SR, Benaron, DA, Van Houten, JP, Duckworth, JL, Liu, FWH, Spilman, SD, Stevenson, DK and Cheong, WF, Stationary headband for clinical time-of-flight optical imaging at the bedside, Photochemistry and Photobiology, 1998, 68, 361-369.

[127] Schmidt, F. E. W., Fry, M. E., Hillman, E. M. C., Hebden, J. C. and Delpy, D. T., A 32-channel timeresolved instrument for medical optical tomography, Review of Scientific Instruments, 2000, 71(1), 256-265. 
[128] Hebden, J. C., Gibson, A., Yusof, R. M., Everdell, N., Hillman, E. M. C., Delpy, D. T., Arridge, S. R., Austin, T., Meek, J. H. and Wyatt, J. S., Three-dimensional optical tomography of the premature infant brain, Physics in Medicine and Biology, 2002, 47(23), 4155-4166.

[129] Hebden, JC, Price, BD, Gibson, AP and Royle, G, A soft phantom for diffuse optical tomography, Physics in Medicine and Biology, 2006, 51, 5581-5590.

[130] Price, BD, Gibson, AP, Tan, LT and Royle, GJ, An elastically compressible phantom material, Physics in Medicine and Biology, 2010, 55, 1177-1188.

[131] Gibson, AP, Austin, T, Everdell, NL, Schweiger, M, Arridge, SR, Meek, JH, Wyatt, JS, Delpy, DT and Hebden, JC, Three-dimensional whole-head optical tomography of passive motor evoked responses in the neonate, Neuroimage, 2006, 30(2), 521-528.

[132] Lloyd-Fox, S., Blasi, A. and Elwell, C. E., Illuminating the developing brain: The past, present and future of functional near infrared spectroscopy, Neuroscience and Biobehavioral Reviews, 2010, 34(3), 269-284.

[133] Minagawa-Kawai, Y, Mori, K, Hebden, JC and Dupoux, E, Optical imaging of infants' neurocognitive development: Recent advances and perspectives, Developmental Neurobiology, 2008, 68(6), 712-728.

[134] Lin, PY, Lin, SI, Penney, T and Chen, JJJ, Review: Applications of Near Infrared Spectroscopy and Imaging for Motor Rehabilitation in Stroke Patients, Journal of Medical and Biological Engineering, 2009, 29(5).

[135] Hebden, J. C. and Austin, T., Optical tomography of the neonatal brain, European Radiology, 2007, 17(11), 2926-2933.

[136] Maris, M, Gratton, E, Maier, J, Mantulin, W and Chance, B, Functional near - infrared imaging of deoxygenated hemoglobin during exercise of the finger extensor muscles using the frequency - domain technique, Bioimaging, 1994, 2(4), 174-183.

[137] Graber, HL, Zheng, S, Pei, Y, Schmitz, CH, Arif, I, Hira, J and Barbour, RL, ed., Dynamic imaging of muscle activity by optical tomography, 2000, 407-408.

[138] Hillman, EMC, Hebden, JC, Schweiger, M, Dehghani, H, Schmidt, FEW, Delpy, DT and Arridge, SR, Time resolved optical tomography of the forearm, Physics in Medicine and Biology, 2001, 46, $1117-1130$.

[139] Zhao, H, Gao, F, Tanikawa, Y, Homma, K and Yamada, Y, Time-resolved diffuse optical tomographic imaging for the provision of both anatomical and functional information about biological tissue, Applied Optics, 2005, 44(10), 1905-1916.

[140] Klose, A., Prapavat, V., Minet, O., Beuthan, J. and Muller, G., Investigations of RA-diagnostics applying optical tomography in frequency-domain, Optical and Imaging Techniques for Biomonitoring Iii, Proceedings Of, 1998, 3196, 194-205.

[141] Hielscher, A. H., Klose, A. D., Scheel, A. K., Moa-Anderson, B., Backhaus, M., Netz, U. and Beuthan, J., Sagittal laser optical tomography for imaging of rheumatoid finger joints, Physics in Medicine and Biology, 2004, 49(7), 1147-1163.

[142] Scheel, A. K., Backhaus, M., Klose, A. D., Moa-Anderson, B., Netz, U. J., Hermann, K. G. A., Beuthan, J., Muller, G. A., Burmester, G. R. and Hielscher, A. H., First clinical evaluation of sagittal laser optical tomography for detection of synovitis in arthritic finger joints, Annals of the Rheumatic Diseases, 2005, 64(2), 239-245.

[143] Hielscher, A. H., Lasker, J. M., Fong, C. J. and Dwyer, E., Imaging of Hemodynamic effects in arthritic joints with dynamic optical tomography Diffuse Optical Imaging of Tissue, 2007, 6629, 6290.

[144] Wang, X., Chamberland, D. L., Fowlkes, J. B., Carson, P. L. and Jamadar, D. A., Photoacoustic tomography of small-animal and human peripheral joints Photons Plus Ultrasound: Imaging and Sensing 2008: the Ninth Conference on Biomedical Thermoacoustics, Optoacoustics, and AcousticOptics, 2008, 6856, 85604 . 
[145] Chamberland, D. L., Agarwal, A., Kotov, N., Fowlkes, J. B., Carson, P. L. and Wang, X., Photoacoustic tomography of joints aided by an Etanercept-conjugated gold nanoparticle contrast agent - an ex vivo preliminary rat study, Nanotechnology, 2008, 19(9), - .

[146] Pardo-Martin, C., Pengo, T., Munoz-Barrutia, A. and Ortiz-De-Solorzano, C., Photon migration simulator for fluorescence tomography - art. no. 69130B, 2008, B9130-B9130.

[147] Zhao, H. J., Zhang, S. Q., Wang, Z. X., Miao, H., Du, Z. and Jiang, J. Y., Frequency-domain inverse Monte Carlo simulation for the diagnosis of the early cervical cancer based on NIR diffuse measurement Imaging, Manipulation, and Analysis of Biomolecules, Cells, and Tissues Vi, 2008, 6859, N8591-N8591, 251.

[148] Xia, Z., Zhou, X. B., Sun, Y. X. and Wong, S. T. C., Registration of 3D FMT and CT images of mouse via affine transformation using Sequential Monte Carlo, Computational Models for Life Sciences (Cmls 07), 2007, 952, 48-56.

[149] Ma, G. B., Delorme, J. F., Gallant, P. and Boas, D. A., Comparison of simplified Monte Carlo simulation and diffusion approximation for the fluorescence signal from phantoms with typical mouse tissue optical properties, Applied Optics, 2007, 46(10), 1686-1692.

[150] Wang, D. F., Liu, X., Chen, Y. P. and Bai, J., A Novel Finite-Element-Based Algorithm for Fluorescence Molecular Tomography of Heterogeneous Media, IEEE Transactions on Information Technology in Biomedicine, 2009, 13(5), 766-773.

[151] Joshi, A., Rasmussen, J. C., Sevick-Muraca, E. M., Wareing, T. A. and McGhee, J., Radiative transport-based frequency-domain fluorescence tomography, Physics in Medicine and Biology, 2008, 53(8), 2069-2088.

[152] Herve, L., Da Silva, A., Boutet, J., Frassati, A., Dinten, J. M., Peltie, P. and Rizo, P., Non contact fluorescence optical tomography by means of numerical and analytical approaches, Nuclear Instruments \& Methods in Physics Research Section a-Accelerators Spectrometers Detectors and Associated Equipment, 2007, 571(1-2), 203-206.

[153] Ntziachristos, V., Fluorescence molecular imaging, Annual Review of Biomedical Engineering, 2006, $8,1-33$.

[154] Zhou, L, Yazici, B and Ntziachristos, V, ed., Fluorescence molecular-tomography reconstruction with a priori anatomical information, 2008, 686800-1.

[155] Tan, Y. Y. and Jiang, H. B., Diffuse optical tomography guided quantitative fluorescence molecular tomography, Applied Optics, 2008, 47(12), 2011-2016.

[156] Hyde, D, Soubret, A, Dunham, J, Lasser, T, Miller, E, Brooks, D and Ntziachristos, V, ed., Analysis of reconstructions in full view fluorescence molecular tomography, 2007, 649803.

[157] Garofalakis, A., Zacharakis, G., Meyer, H., Economou, E. N., Mamalaki, C., Papamatheakis, J., Kioussis, D., Ntziachristos, V. and Ripoll, J., Three-dimensional in vivo imaging of green fluorescent protein-expressing $\mathrm{T}$ cells in mice with noncontact fluorescence molecular tomography, Molecular Imaging, 2007, 6(2), 96-107.

[158] Janunts, E, P schinger, T, Brünner, H and Langenbucher, A, Linear method of fluorescent source reconstruction in a diffusion medium, Zeitschrift Fur Medizinische Physik, 2008, 18(3), 189-196.

[159] Hayashi, T, Kashio, Y and Okada, E, Hybrid Monte Carlo-diffusion method for light propagation in tissue with a low-scattering region, Applied Optics, 2003, 42(16), 2888-2896.

[160] Koyama, T, Iwasaki, A, Ogoshi, Y and Okada, E, Practical and adequate approach to modeling light propagation in an adult head with low-scattering regions by use of diffusion theory, Applied Optics, 2005, 44(11), 2094-2103.

[161] Tarvainen, T, Vauhkonen, M, Kolehmainen, V and Kaipio, JP, Finite element model for the coupled radiative transfer equation and diffusion approximation, International Journal for Numerical Methods in Engineering, 2006, 65(3), 383-405. 
[162] Tarvainen, T, Vauhkonen, M, Kolehmainen, V and Kaipio, JP, Hybrid radiative-transfer-diffusion model for optical tomography, Applied Optics, 2005, 44(6), 876-886.

[163] Roy, R, ed., Telegrapher-based fluorescence-enhanced optical tomography in small volume, 2010, $75610 \mathrm{H}$.

[164] Song, X. L., Wang, D. F., Chen, N. G., Bai, J. and Wang, H., Reconstruction for free-space fluorescence tomography using a novel hybrid adaptive finite element algorithm, Optics Express, 2007, 15(26), 18300-18317.

[165] Roy, R., Godavarty, A., Thompson, A. B. and Sevick-Muraca, E. M., Image reconstruction for diagnosis and prognosis of breast cancer using fluorescence measurements: Phantom studies, Optical Tomography and Spectroscopy of Tissue VI, 2005, 5693, 203-209.

[166] Jun, Z., Zhang, Y. H. and Jing, B., Investigation of continuous wave near infrared optical tomography based on gradient optimization schemes, Journal of Infrared and Millimeter Waves, 2004, 23(3), 161-+.

[167] Hielscher, A. H. and Bartel, S., Parallel programming of gradient-based iterative image reconstruction schemes for optical tomography, Computer Methods and Programs in Biomedicine, 2004, 73(2), 101-113.

[168] Roy, R. and Sevick-Muraca, E. M., A numerical study of gradient-based nonlinear optimization methods for contrast enhanced optical tomography, Optics Express, 2001, 9(1), 49-65.

[169] Roy, R, ed., Numerical comparison of different penalty modified barrier functions for optical tomography problems, 2009, $71740 \mathrm{Z}$.

[170] Roy, R., Godavarty, A., Thompson, A. B. and Sevick-Muraca, E. M., Penalty/modified barrier function method for diagnostic imaging using area and point illumination geometries in fluorescence-enhanced optical tomography, 2004.

[171] Roy, R., Thompson, A. B., Godavarty, A. and Sevick-Muraca, E. M., Tomographic fluorescence imaging in tissue phantoms: A novel reconstruction algorithm and imaging geometry, IEEE Transactions on Medical Imaging, 2005, 24(2), 137-154.

[172] Ntziachristos, V. and Weissleder, R., Experimental three-dimensional fluorescence reconstruction of diffuse media by use of a normalized Born approximation, Optics Letters, 2001, 26(12), 893-895.

[173] Graves, E. E., Ripoll, J., Weissleder, R. and Ntziachristos, V., A submillimeter resolution fluorescence molecular imaging system for small animal imaging, Medical Physics, 2003, 30(5), 901-911.

[174] Song, X. L. and Jing, B., An improved finite-element based reconstruction algorithm for fluorescence tomography, Cisp 2008: First International Congress on Image and Signal Processing, Vol 3, Proceedings, 2008, 761-765.

[175] Roy, R and Sevick-Muraca, E, Truncated Newton's optimization scheme for absorption and fluorescence optical tomography: Part I theory and formulation, Optics Express, 1999, 4(10), 353-371.

[176] Eppstein, MJ, Hawrysz, DJ, Godavarty, A and Sevick-Muraca, EM, Three-dimensional, Bayesian image reconstruction from sparse and noisy data sets: near-infrared fluorescence tomography, Proceedings of the National Academy of Sciences, 2002, 99(15), 9619.

[177] Joshi, A, Bangerth, W and Sevick, E, Non-contact fluorescence optical tomography with adaptive finite element methods, Mathematical Methods in Biomedical Imaging and Intensity-Modulated Radiation Therapy (IMRT), Y. Censor, M. Jiang, and AK Louis, Eds. Birkh user, 2008.

[178] Xu, Z. and Bai, J., Analysis of finite-element-based methods for reducing the ill-posedness in the reconstruction of fluorescence molecular tomography, Progress in Natural Science, 2009, 19(4), $501-509$.

[179] Zacharopoulos, A. D., Svenmarker, P., Axelsson, J., Schweiger, M., Arridge, S. R. and AnderssonEngels, S., A matrix-free algorithm for multiple wavelength fluorescence tomography, Optics Express, 2009, 17(5), 3025-3035.

[180] Hervé, L, Koenig, A, Da Silva, A, Berger, M, Boutet, J, Dinten, JM, Peltié, P and Rizo, P, Noncontact fluorescence diffuse optical tomography of heterogeneous media, Applied Optics, 2007, 46(22), 4896-4906. 
[181] Cheng, X. F. and Boas, D. A., Systematic diffuse optical image errors resulting from uncertainty in the background optical properties, Optics Express, 1999, 4(8), 299-307.

[182] Mohajerani, P., Eftekhar, A. A. and Adibi, A., Object localization in the presence of a strong heterogeneous background in fluorescent tomography, Journal of the Optical Society of America aOptics Image Science and Vision, 2008, 25(6), 1467-1479.

[183] Herve, L., Koenig, A., Da Silva, A., Berger, M., Boutet, J., Peltie, P., Rizo, P. and Dinten, J. M., Algorithm for in vivo $\mathrm{cw}$ fluorescence diffuse optical tomography taking into account optical heterogeneities of biological tissues and the shape of the animal, 2007 4th IEEE International Symposium on Biomedical Imaging : Macro to Nano, 2007, 1-3, 812-815.

[184] Tan, Y. Y. and Jiang, H. B., DOT guided fluorescence molecular tomography of arbitrarily shaped objects, Medical Physics, 2008, 35(12), 5703-5707.

[185] Bartels, M., Joshi, A., Rasmussen, J. C., Bangerth, W. and Sevick-Muraca, E. M., Post image acquisition mitigation of excitation light leakage in patterned illumination based NIR fluorescence tomography, 2008 IEEE International Symposium on Biomedical Imaging: from Nano to Macro, Vols $1-4,2008,1239-1242$.

[186] Hwang, K., Houston, J. P., Rasmussen, J. C., Ke, S., Li, C. and Sevick-Muraca, E. M., The influence of improved interference filter performance for molecular imaging using frequency domain photon migration measurements, Optical Tomography and Spectroscopy of Tissue VI, 2005, 5693, 503-512, 538 .

[187] Hwang, K., Pan, T., Joshi, A., Rasmussen, J. C., Bangerth, W. and Sevick-Muraca, E. M., Influence of excitation light rejection on forward model mismatch in optical tomography, Physics in Medicine and Biology, 2006, 51(22), 5889-5902.

[188] Kepshire, DL, Dehghani, H, Leblond, F and Pogue, BW, Automatic exposure control and estimation of effective system noise in diffuse fluorescence tomography, Opt. Express, 2009, 17, 23272-23283.

[189] Soloviev, V. Y., McGinty, J., Tahir, K. B., Neil, M. A. A., Sardini, A., Hajnal, J. V., Arridge, S. R. and French, P. M. W., Fluorescence lifetime tomography of live cells expressing enhanced green fluorescent protein embedded in a scattering medium exhibiting background autofluorescence, Optics Letters, 2007, 32(14), 2034-2036.

[190] Soubret, A. and Ntziachristos, V., Fluorescence molecular tomography in the presence of background fluorescence, Physics in Medicine and Biology, 2006, 51(16), 3983-4001.

[191] Gao, M., Lewis, G., Turner, G. M., Soubret, A. and Ntziachristos, V., Effects of background fluorescence in fluorescence molecular tomography, Applied Optics, 2005, 44(26), 5468-5474.

[192] Zhu, B., Sevick-Muraca, E. M., Eppstein, M. J. and Godavarty, A., Noise filtration in fluorescenceenhanced optical tomography: breast phantom studies, Inverse Problems in Science and Engineering, 2009, 17(1), 97-104.

[193] Zhu, B., Eppstein, M. J., Sevick-Muraca, E. M. and Godavarty, A., Noise pre-filtering techniques in fluorescence-enhanced optical tomography, Optics Express, 2007, 15(18), 11285-11300.

[194] Psycharakis, S., Zacharakis, G., Garofalakis, A., Favicchio, R. and Ripoll, J., Autofluorescence removal from fluorescence tomography data using multispectral imaging, Molecular Imaging, 2007, 6626, U71-U77.

[195] Lin, Y., Gao, H., Nalcioglu, O. and Gulsen, G., Fluorescence diffuse optical tomography with functional and anatomical a priori information: feasibility study, Physics in Medicine and Biology, 2007, 52(18), 5569-5585.

[196] Zhou, L., Yazici, B. and Ntziachristos, V., Fluorescence molecular-tomography reconstruction with a priori anatomical information, Small Animal Whole-Body Optical Imaging Based on Genetically Engineered Probes, 2008, 6868, O8680-08680.

[197] Hyde, D., Schulz, R., Brooks, D., Miller, E. and Ntziachristos, V., Performance dependence of hybrid x-ray computed tomography/fluorescence molecular tomography on the optical forward problem, Journal of the Optical Society of America a-Optics Image Science and Vision, 2009, 26(4), 919-923. 
[198] Cho, W. S., Cho, M. J., Kim, S. R., Choi, M., Lee, J. Y., Han, B. S., Park, S. N., Yu, M. K., Jon, S. and Jeong, J., Pulmonary toxicity and kinetic study of Cy5.5-conjugated superparamagnetic iron oxide nanoparticles by optical imaging, Toxicology and Applied Pharmacology, 2009, 239(1), 106-115.

[199] Xu, H., Eck, P. K., Baidoo, K. E., Choyke, P. L. and Brechbiel, M. W., Toward preparation of antibodybased imaging probe libraries for dual-modality positron emission tomography and fluorescence imaging, Bioorganic \& Medicinal Chemistry, 2009, 17(14), 5176-5181.

[200] Zhu, Q, Cronin, EB, Currier, AA, Vine, HS, Huang, M, Chen, NG and Xu, C, Benign versus Malignant Breast Masses: Optical Differentiation with US-guided Optical Imaging Reconstruction1, Radiology, 2005, 237(1), 57.

[201] Godavarty, A., Thompson, A. B., Roy, R., Gurfinkel, M., Eppstein, M. J., Zhang, C. and SevickMuraca, E. M., Diagnostic imaging of breast cancer using fluorescence-enhanced optical tomography: phantom studies, Journal of Biomedical Optics, 2004, 9(3), 488-496.

[202] Patwardhan, S. V., Bloch, S. R., Achilefu, S. and Culver, J. P., Time-dependent whole-body fluorescence tomography of probe bio-distributions in mice, Optics Express, 2005, 13(7), 2564-2577.

[203] Lam, S., Lesage, F. and Intes, X., Time domain fluorescent diffuse optical tomography: analytical expressions, Optics Express, 2005, 13(7), 2263-2275.

[204] Lam, S., Lesage, F. and Intes, X., Time domain fluorescent diffuse optical tomography, Photonics North: Applications of Photonic Technology 7B, Pts 1 and 2, 2004, 5578, 179-187.

[205] Gao, F., Xue, Y., Zhao, H. J., Tanikawa, Y. and Yamada, Y., A linear GPST-based scheme for timedomain fluorescence molecular tomography Optical Tomography and Spectroscopy of Tissue VII, $2007,6434,4342$.

[206] Gao, F., Zhao, H. J., Zhang, L. M., Tanikawa, Y., Marjono, A. and Yamada, Y., A self-normalized, full time-resolved method for fluorescence diffuse optical tomography, Optics Express, 2008, 16(17), 13104-13121.

[207] Gao, F., Zhang, L. M., Li, J. and Zhao, H. J., Experimental validation for time-domain fluorescence diffuse optical tomography of linear scheme, Chinese Optics Letters, 2008, 6(12), 889-892.

[208] Ntziachristos, V. and Weissleder, R., Charge-coupled-device based scanner for tomography of fluorescent near-infrared probes in turbid media, Medical Physics, 2002, 29(5), 803-809.

[209] Ko, W., Kwak, Y. and Kim, S., Development of a non-contact diffuse optical tomography system for image reconstruction of blood vessel with NIR light, World Congress on Medical Physics and Biomedical Engineering 2006, 2007, 14, 1345-1348.

[210] Hu, G., Yao, J. J. and Bai, J., Full-angle optical imaging of near-infrared fluorescent probes implanted in small animals, Progress in Natural Science, 2008, 18(6), 707-711.

[211] Leblond, F., Davis, S. C., Valdes, P. A. and Pogue, B. W., Pre-clinical whole-body fluorescence imaging: Review of instruments, methods and applications, Journal of Photochemistry and Photobiology B-Biology, 2010, 98(1), 77-94.

[212] Ntziachristos, V., Bremer, C., Tung, C. and Weissleder, R., Imaging cathepsin B up-regulation in HT-1080 tumor models using fluorescence-mediated molecular tomography (FMT), Academic Radiology, 2002, 9, S323-S325.

[213] Ntziachristos, V., Schellenberger, E. A., Ripoll, J., Yessayan, D., Graves, E., Bogdanov, A., Josephson, L. and Weissleder, R., Visualization of antitumor treatment by means of fluorescence molecular tomography with an annexin V-Cy5.5 conjugate, Proceedings of the National Academy of Sciences of the United States of America, 2004, 101(33), 12294-12299.

[214] Montet, X., Ntziachristos, V., Grimm, J. and Weissleder, R., Tomographic fluorescence mapping of tumor targets, Cancer Research, 2005, 65(14), 6330-6336.

[215] Garofalakis, A., Meyer, H., Zacharakis, G., Economou, E. N., Mamalaki, C., Papamatheakis, J., Ntziachristos, V. and Ripoll, J., 3D in-vivo imaging of GFP-expressing T-cells in mice with noncontact fluorescence molecular tomography, Saratov Fall Meeting 2004: Optical Technologies in Biophysics and Medicine VI, 2005, 5771, 120-129. 
[216] Haller, J., Hyde, D., Deliolanis, N., de Kleine, R., Niedre, M. and Ntziachristos, V., Visualization of pulmonary inflammation using noninvasive fluorescence molecular imaging, Journal of Applied Physiology, 2008, 104(3), 795-802.

[217] Deliolanis, N., Lasser, T., Niedre, M., Soubret, A. and Ntziachristos, V., In-vivo lung cancer imaging in mice using 360 degrees free-space fluorescence molecular tomography, 2006 28th Annual International Conference of the IEEE Engineering in Medicine and Biology Society, Vols 1-15, 2006, $2828-2830$.

[218] Hyde, D., de Kleine, R., MacLaurin, S. A., Miller, E., Brooks, D. H., Krucker, T. and Ntziachristos, V., Hybrid FMT-CT imaging of amyloid-beta plaques in a murine Alzheimer's disease model, Neuroimage, 2009, 44(4), 1304-1311.

[219] Nahrendorf, M., Sosnovik, D. E., French, B. A., Swirski, F. K., Bengel, F., Sadeghi, M. M., Lindner, J. R., Wu, J. C., Kraitchman, D. L., Fayad, Z. A. and Sinusas, A. J., Multimodality Cardiovascular Molecular Imaging, Part II, Circulation-Cardiovascular Imaging, 2009, 2(1), 56-70.

[220] Sinusas, A. J., Bengel, F., Nahrendorf, M., Epstein, F. H., Wu, J. C., Villanueva, F. S., Fayad, Z. A. and Gropler, R. J., Multimodality Cardiovascular Molecular Imaging, Part I, Circulation-Cardiovascular Imaging, 2008, 1(3), 244-256.

[221] Hildebrandt, I. J. and Gambhir, S. S., Molecular imaging applications for immunology, Clinical Immunology, 2004, 111(2), 210-224.

[222] Roy, R., Godavarty, A. and Sevick-Muraca, E. M., Fluorescence-enhanced three-dimensional lifetime imaging: a phantom study, Physics in Medicine and Biology, 2007, 52(14), 4155-4170.

[223] Roy, R., Godavarty, A., Thompson, A. B. and Sevick-Muraca, E. M., Image reconstruction for diagnosis and prognosis of breast cancer using fluorescence measurements: Phantom studies, Optical Tomography and Spectroscopy of Tissue VI, 2005, 5693, 203-209.

[224] Godavarty, A., Eppstein, M. J., Zhang, C. Y. and Sevick-Muraca, E. M., Detection of single and multiple targets in tissue phantoms with fluorescence-enhanced optical imaging: Feasibility study, Radiology, 2005, 235(1), 148-154.

[225] Godavarty, A., Zhang, C., Eppstein, M. J. and Sevick-Muraca, E. M., Fluorescence-enhanced optical imaging of large phantoms using single and simultaneous dual point illumination geometries, Medical Physics, 2004, 31(2), 183-190.

[226] Roy, R., Godavarty, A. and Sevick-Muraca, E. M., Fluorescence-enhanced optical tomography using referenced measurements of heterogeneous media, IEEE Transactions on Medical Imaging, 2003, 22(7), 824-836.

[227] Sevick-Muraca, E. M., Houston, J. P. and Gurfinkel, M., Fluorescence-enhanced, near infrared diagnostic imaging with contrast agents, Current Opinion in Chemical Biology, 2002, 6(5), $642-650$.

[228] Davis, S. C., Gibbs-Strauss, S. L., Tuttle, S. B., Jiang, S., Springett, R., Dehghani, H., Pogue, B. W. and Paulsen, K. D., MRI-coupled spectrally resolved fluorescence tomography for in vivo imaging, Proceedings of the SPIE - The International Society for Optical Engineering, 2008, 6850, 9.

[229] McCann, C. M., Waterman, P., Figueiredo, J. L., Aikawa, E., Weissleder, R. and Chen, J. W., Combined magnetic resonance and fluorescence imaging of the living mouse brain reveals glioma response to chemotherapy, Neuroimage, 2009, 45(2), 360-369.

[230] Nahrendorf, M., Waterman, P. and Weissleder, R., Dual Channel Hybrid Fluorescence Molecular Tomography/X-ray Computed Tomography (FMT-CT) of Inflammation in Murine Atherosclerotic Plaques, Circulation, 2008, 118(18), S554-S554.

[231] Willmann, Jurgen K., van Bruggen, Nicholas, Dinkelborg, Ludger M. and Gambhir, Sanjiv S., Molecular imaging in drug development, Nat Rev Drug Discov, 2008, 7(7), 591-607.

[232] Grassi, R., Cavaliere, C., Cozzolino, S., Mansi, L., Cirillo, S., Tedeschi, G., Franchi, R., Russo, P., Cornacchia, S. and Rotondo, A., Small animal imaging facility: new perspectives for the radiologist, Radiologia Medica, 2009, 114(1), 152-167. 
[233] Ripoll, J., Ntziachristos, V., Cannet, C., Babin, A. L., Kneuer, R., Gremlich, H. U. and Beckmann, N., Investigating pharmacology in vivo using magnetic resonance and optical imaging, Drugs in $R \& D$, 2008, 9(5), 277-306.

[234] Hoshino, H., Current advanced bioluminescence technology in drug discovery, Expert Opinion on Drug Discovery, 2009, 4(4), 373-389.

[235] Montet, X., Figueiredo, J. L., Alencar, H., Ntziachristos, V., Mahmood, U. and Weissleder, R., Tomographic fluorescence imaging of tumor vascular volume in mice, Radiology, 2007, 242(3), 751-758.

[236] Qian, H. M., Gu, Y. Q., Wang, M. and Achilefu, S., Optimization of the Near-Infrared Fluorescence Labeling for In Vivo Monitoring of a Protein Drug Distribution in Animal Model, Journal of Fluorescence, 2009, 19(2), 277-284.

[237] Da Silva, A., Dinten, J. M., Peltie, P., Coll, J. L. and Rizo, P., In vivo fluorescence molecular optical imaging: from small animal towards clinical applications, 2008 Mediterranean Conference on Control Automation, 2008, 1-4, 459-464.

[238] Nuiachristos, V., Fluorescence molecular imaging, Annual Review of Biomedical Engineering, 2006, 8, 1-33.

[239] Ntziachristos, V., Graves, E., Schultz, R. F. and Ripoll, J., Fluorescence Molecular Tomography: New detection schemes for acquiring high information content measurements, 2004 2Nd IEEE International Symposium on Biomedical Imaging: Macro to Nano, 2004, 1-2, 1475-1478.

[240] Gibson, A. and Dehghani, H., Diffuse optical imaging, Philosophical Transactions of the Royal Society a-Mathematical Physical and Engineering Sciences, 2009, 367(1900), 3055-3072.

[241] Tian, J., Bai, J., Yan, X. P., Bao, S. L., Li, Y. H., Liang, W. and Yang, X., Multimodality molecular imaging - Improving image quality, IEEE Engineering in Medicine and Biology Magazine, 2008, 27(5), 48-57.

[242] Kaijzel, E. L., Snoeks, T. J. A., Buijs, J. T., van der Pluijm, G. and Lowik, C. W. G. M., Multimodal imaging and treatment of bone metastasis, Clinical \& Experimental Metastasis, 2009, 26(4), 371-379.

[243] Jaffer, F. A., Libby, P. and Weissleder, R., Optical and Multimodality Molecular Imaging Insights Into Atherosclerosis, Arteriosclerosis Thrombosis and Vascular Biology, 2009, 29(7), 1017-U98.

[244] Calef, U, BUSINESS OFFICE FEATURE: Life Science Technologies: Molecular Imaging--No Swiss Army Approach, Science, 2009, 323(5919), 1364.

[245] Rousseau, G, Blouin, A and Monchalin, JP, ed., Spatial resolution of ultrasound-modulated optical tomography used for the detection of absorbing and scattering objects in thick scattering media, 2010, 75642A.

[246] Brooksby, B, Pogue, BW, Jiang, S, Dehghani, H, Srinivasan, S, Kogel, C, Tosteson, TD, Weaver, J, Poplack, SP and Paulsen, KD, Imaging breast adipose and fibroglandular tissue molecular signatures by using hybrid MRI-guided near-infrared spectral tomography, Proceedings of the National Academy of Sciences, 2006, 103(23), 8828.

[247] Boverman, G., Fang, Q., Carp, S. A., Miller, E. L., Brooks, D. H., Selb, J., Moore, R. H., Kopans, D. B. and Boas, D. A., Spatio-temporal imaging of the hemoglobin in the compressed breast with diffuse optical tomography, Physics in Medicine and Biology, 2007, 52(12), 3619-3641.

[248] Davis, S. C., Gibbs-Strauss, S. L., Tuttle, S. B., Jiang, S., Springett, R., Dehghani, H., Pogue, B. W. and Paulsen, K. D., MRI-Coupled spectrally-resolved fluorescence tomography for in vivo Imaging, Multimodal Biomedical Imaging Iii, 2008, 6850, 8500.

[249] Hyde, D, de Kleine, R, MacLaurin, SA, Miller, E, Brooks, DH, Krucker, T and Ntziachristos, V, Hybrid FMT-CT imaging of amyloid-[beta] plaques in a murine Alzheimer's disease model, Neuroimage, 2009, 44(4), 1304-1311. 
[250] Nahrendorf, M, Waterman, P, Thurber, G, Groves, K, Rajopadhye, M, Panizzi, P, Marinelli, B, Aikawa, E, Pittet, MJ and Swirski, FK, Hybrid in vivo FMT-CT imaging of protease activity in atherosclerosis with customized nanosensors, Arteriosclerosis, thrombosis, and vascular biology, 2009.

[251] Paudyal, P., Paudyal, B., Iida, Y., Oriuchi, N., Hanaoka, H., Tominaga, H., Ishikita, T., Yoshioka, H., Higuchi, T. and Endo, K., Dual functional molecular imaging probe targeting CD20 with PET and optical imaging, Oncology Reports, 2009, 22(1), 115-119.

[252] Razansky, D., Vinegoni, C. and Ntziachristos, V., Multi-spectral photo-acoustic molecular tomography resolves fluorochrome distribution with high resolution and sensitivity in small animals Photons Plus Ultrasound: Imaging and Sensing 2008: the Ninth Conference on Biomedical Thermoacoustics, Optoacoustics, and Acoustic-Optics, 2008, 6856, 85613-85613. 


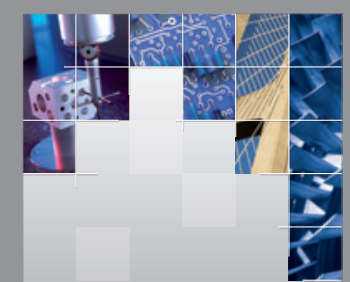

\section{Enfincering}
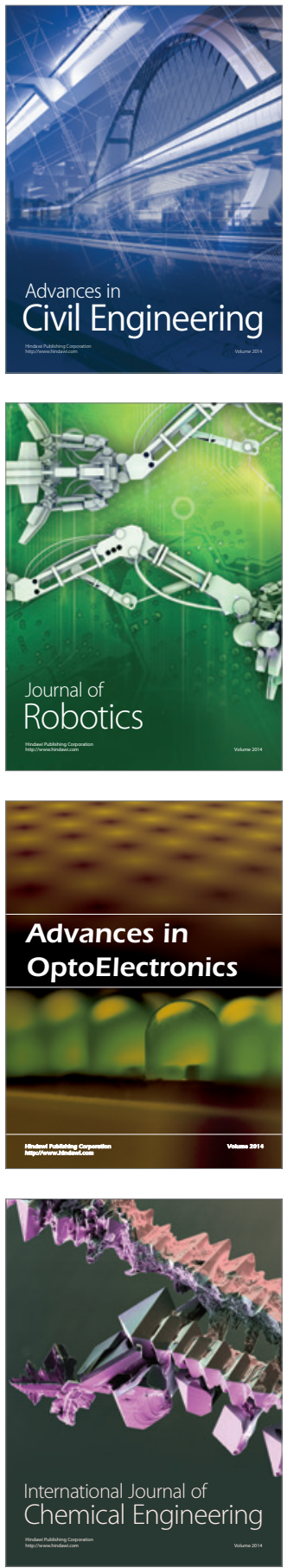

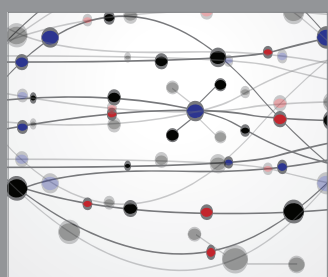

The Scientific World Journal

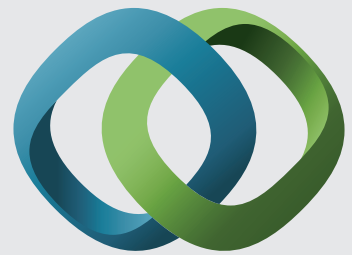

\section{Hindawi}

Submit your manuscripts at

http://www.hindawi.com
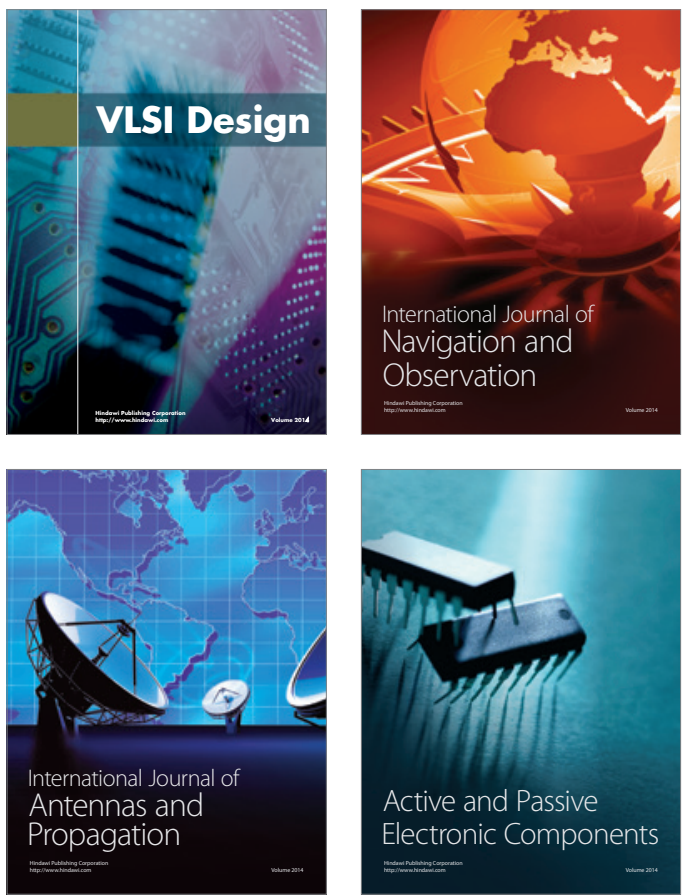
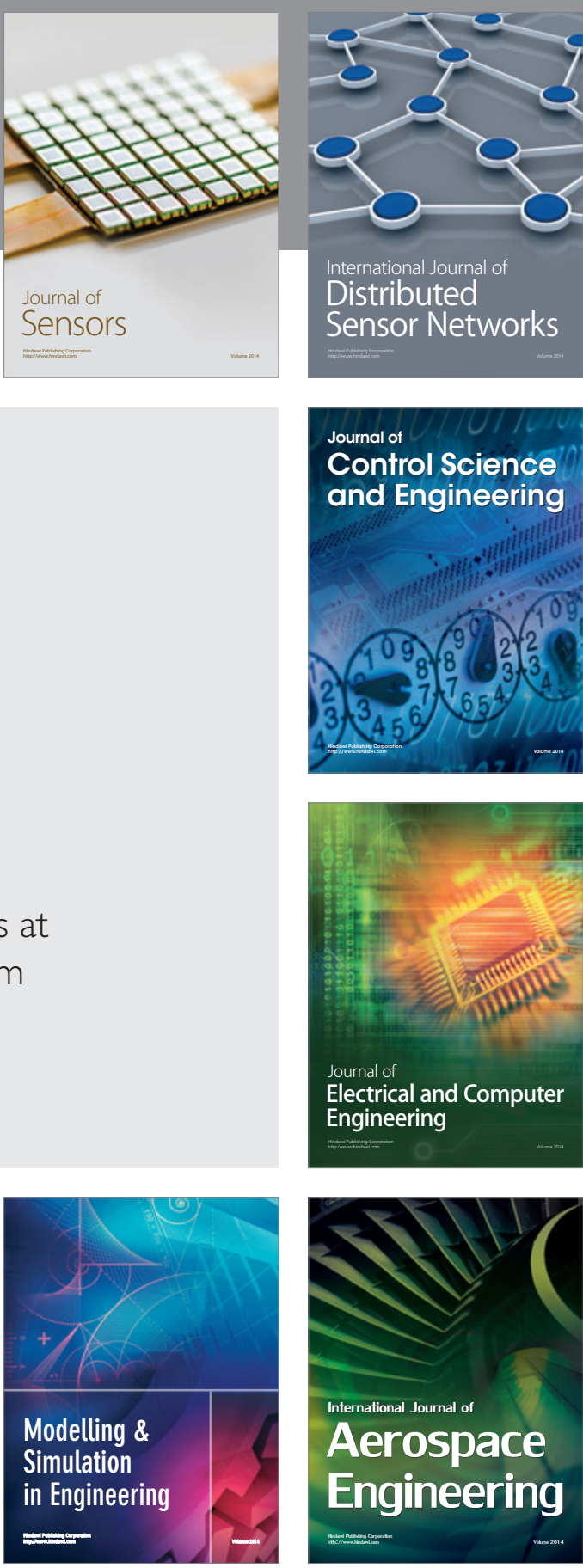

International Journal of

Distributed

Sensor Networks

Journal of

Control Science

and Engineering
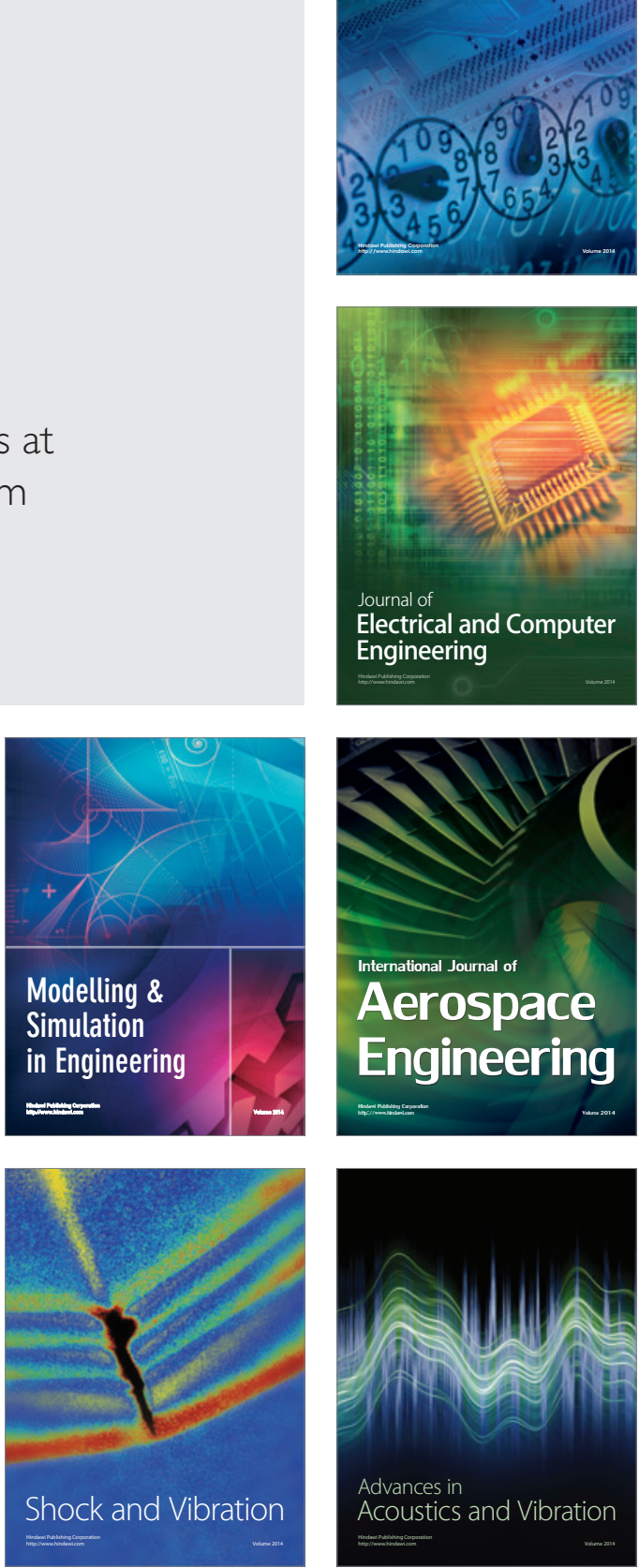